\title{
Development of a Powerful Synthetic Hybrid Promoter to Improve the Cellulase System of Trichoderma Reesei for Efficient Saccharification of Corncob Residues
}

\section{Yifan Wang}

Institute of Microbial Technology, Shandong University

\section{Ruiyan Liu}

Institute of Microbial Technology, Shandong University

\section{Hong Liu}

Institute of Microbial Technology, Shandong University

Xihai Li

Institute of Microbial Technology, Shandong University

Linjing Shen

Institute of Microbial Technology, Shandong University

\section{Weican Zhang}

Institute of Microbial Technology, Shandong University

\section{Xin Song}

Institute of Microbial Technology, Shandong University

\section{Weifeng Liu}

Institute of Microbial Technology, Shandong University

Xiangmei Liu

Institute of Microbial Technology, Shandong University

Yaohua Zhong ( $\nabla$ zhongyaohua@sdu.edu.cn )

Institute of Microbial Technology $₫$ Shandong University

\section{Research Article}

Keywords: Synthetic hybrid promoter, Trichoderma reesei, cbh1, cdna1, Cellulase, Biomass bioconversion

Posted Date: June 9th, 2021

DOI: https://doi.org/10.21203/rs.3.rs-584234/v1

License: (c) (i) This work is licensed under a Creative Commons Attribution 4.0 International License. Read Full License

Version of Record: A version of this preprint was published at Microbial Cell Factories on January 4th, 2022. See the published version at https://doi.org/10.1186/s12934-021-01727-8. 


\section{Abstract}

Background: The filamentous fungus Trichoderma reesei is a widely used workhorse for cellulase production in industry due to its prominent secretion capacity of extracellular cellulolytic enzymes. However, some key components are not always sufficient in this cellulase cocktail, making the conversion of cellulose-based biomass costly on the industrial scale. Development of strong and efficient promoters would enable cellulase cocktail to be optimized for bioconversion of biomass.

Results: In this study, a synthetic hybrid promoter was constructed and applied to optimize the cellulolytic system of $T$. reesei for efficient saccharification towards corncob residues. Firstly, a promoter library was established by sequence truncation based on the strong constitutive promoter Pcdna1. The strongest promoter amongst them was Pcdna1-3, exhibiting a 1.4-fold higher activity than that of the native cdna 1 promoter. Meanwhile, the activation region (-821 to -622 bp upstream of the translation initiation codon ATG and devoid of the Cre1-binding sites) of the strong inducible promoter Pcbh1 was cloned and identified to be an amplifier in initiating gene expression. Finally, this activation region was fused to the strongest promoter Pcdna 1-3, generating the novel synthetic hybrid promoter Pcc. This engineered promoter Pcc drove strong gene expression by displaying 1.6- and 1.8fold stronger fluorescence intensity than $\mathrm{P} c b h 1$ and $\mathrm{P} c d n a 1$ under the inducible condition using egfp as the reporter gene, respectively. Furthermore, Pcc was applied to overexpress the Aspergillus niger $\beta$-glucosidase BGLA coding gene $b g / A$ and the native endoglucanase EG2 coding gene eg2, achieving a 43.5-fold BGL activity and 1.2-fold EG activity increase, respectively. Ultimately, to overcome the defects of the native cellulase system in T. reesei, the $b g / A$ and eg2 were co-overexpressed under the control of Pcc promoter. The bgIA-eg2 double expression strain QPEB70 exhibited a 178\% increase in total cellulase activity, whose cellulase system displayed 2.3- and 2.4-fold higher saccharification efficiency towards acid-pretreated and delignified corncob residues than the parental strain, respectively.

Conclusions: The synthetic hybrid promoter Pcc was generated and employed to improve the cellulase system of $T$. reesei by expressing specific components. Therefore, construction of synthetic hybrid promoters would allow particular cellulase genes to be expressed at desired levels, which is a viable strategy to optimize the cellulolytic enzyme system for efficient biomass bioconversion.

\section{Introduction}

The filamentous fungus Trichoderma reesei has been widely used as an industrial workhorse for cellulase production due to its extraordinary ability to produce large amounts of extracellular cellulolytic enzymes [1]. Among the cellulase enzymes secreted by this fungus, the cellobiohydrolase $1(\mathrm{CBH} 1)$ accounts for more than $60 \%$ of total extracellular protein [2], thus the $c b h 1$ promoter (Pcbh1) was generally used as a strong inducible promoter for gene expression [3, 4]. Besides, the endoglucanases (EGs) hydrolyzing amorphous cellulose by attacking the cellulose chains internally and randomly is found to make up 6-20\% of the extracellular protein $[5,6]$. Particularly, the $\beta$-glucosidase (BGL) occupying only $1 \%$ of the total extracellular protein was recognized as one of bottlenecks in the process of efficiently hydrolyzing cellulosic substrates [7, 8]. Therefore, this secretome of $T$. reesei is not always a well-proportioned cocktail for the economically feasible bioconversion of lignocellulosic biomass to produce biofuels and other bio-based products [9]. It is known that promoter is essential to both protein and metabolic engineering by controlling gene expression level. In this case, it is prospective to optimize the cellulolytic enzyme system by increasing target gene expression using desired promoters in T. reesei. Indeed, suitable promoters have enabled desired expression levels of the specific cellulase genes, making $T$. reesei exhibit great potential to be cellulase hyper-producing strains $[3,10,11]$. Consequently, appropriate and robust promoters could offer great opportunities for the viable bioconversion of lignocellulosic biomass by conferring high levels of gene expression on specific enzymes to optimize cellulolytic enzyme system.

In $T$. reesei, many native constitutive promoters that can drive constant gene expression were used for protein production, such as promoters of the genes $g p d A$ (encoding glyceraldehyde-3-phosphate dehydrogenase), $p d c$ (encoding pyruvate decarboxylase), eno (encoding enolase), tef (encoding translation elongation factor 1a), and an unidentified gene cdna1 [12, 13, 14]. Among these, Pcdna1 was generally seemed as one of the strongest constitutive promoters reported so far in T. reesei [15]. However, the productivity of protein expressed by Pcdna 1 was not as much as that of the inducible promoter Pcbh1 [14]. In T. reesei, Pcbh1 was recognized as the most widely used promoter for protein production. And the glucose repression regions of Pcbh1 were well characterized [16]. Besides, the binding sites of the transcriptional activators Xyr1, Ace2 and Hap2/3/5, as well as the repressors

Page $2 / 23$ 
Cre1 and Ace1, have been found to exist in the regulation region of Pcbh1 [17-21]. Especially, Cre1 inhibits the gene expression through carbon catabolite repression (CCR) in the presence of easily metabolized carbon source, such as glucose, and serves as one of the master repressors [17]. In this case, deletion of Cre1-binding sites, which is one of the promoter modifications, is an efficient way to enhance the activity of promoter Pcbh1. For example, four-tandem copies of the regulation region of Pcbh1, which lacks the Cre1-binding sites but comprises the Xyr1-binding site, Ace2-binding site and the CCAAT boxes (the Hap2/3/5-binding sites), could serve as transcriptional amplifiers [22]. In addition, the replacement of the Cre1-binding sites by the binding sites of the transcriptional activators Ace2 and Hap2/3/5 also resulted a 5.5-fold Pcbh1 activity increase [21]. However, while the modified regulation region of $\mathrm{Pcbh} 1$ could act as an enhancer in initiating gene expression, the core region usually directs low basal activity when the regulation region is absent [23].

Beyond altering transcription factor binding sites, another choice to modify promoters is hybrid promoter construction [10]. This approach attempts to fuse two independent promoter activation sequences, usually the activation region to the core region. It is known that the cis-elements in promoters are modular and most of them retain their activities when broken away from the natural context, which enables the chimera to be constructed successfully [24]. In the nonconventional yeast Yarrowia lipolytica, a series copy of an upstream activation sequence (UAS) of the endogenous inducible promoter $\mathrm{P}_{\mathrm{XPR} 2}$ were fused to the core region of the constitutive promoter $\mathrm{P}_{\text {LEUM }}$, and in doing so the most efficient one amongst these hybrid promoters led to an eight-fold-higher promoter strength increase compared to the strong endogenous promoters [25]. In addition, the UAS of the inducible promoter $P_{G A L}$ in Saccharomyces cerevisiae was fused to the different core region of constitutive promoters, $P_{T E F}, P_{G P D}, P_{C Y C}$ and $P_{L E U M}$, respectively, and the hybrid promoters induced by galactose exhibited higher activity than that under the glucose condition [26]. These investigations indicated that the synthetic hybrid promoters could offer enormous potential in optimally modifying promoter. Although the synthetic hybrid promoters were extensively used as a potential approach to improve promoters, it is still less commonly used in $T$. reesei [10].

During the process of cellulase hydrolysis, synergistic effects were considered to exist between the independent enzyme components, thus the ratio of each component in the total enzyme cocktail plays an important role in cellulolytic efficiency [27, 28]. Therefore, altering specific enzyme component and amending cellulase mixture to a propriate ratio are efficient approaches to optimize the cellulase system in hydrolyzing lignocellulose [29, 30]. In T. reesei, BGL was generally recognized as one of the targets to be overexpressed due to its lower proportion in optimizing the cellulase system [31, 32]. Introduction of heterogenous BGL from Aspergillus and Penicillium species directed by promoter Pcbh1 enabled the significant increase of cellulase activity and approximately $20 \%$ augment of released glucose in saccharification towards substrates corncob residue and cornstalk, respectively $[29,33]$. Furthermore, extra expression of endogenous BGL directed by a modified four-copy promoter of Pcbh1 led to at most $130 \%$ increment of filter paper activity and $29 \%$ of saccharification efficiency [32]. Besides, EG2 contributes $55 \%$ of the EG activity and when overexpressed in $T$. reesei even use its native promoter, a significant enhancement of lignocellulose hydrolyzing ability $(119 \%$ toward corncob residues) was found [34,35]. Therefore, optimization of $T$. reesei cellulase system by overexpressing specific essential enzymes directed by powerful promoters is a promising solution to improve the lignocellulose hydrolysis efficiency.

In this study, a novel synthetic hybrid promoter Pcc was constructed by fusing the modified activation region of the strongest inducible promoter $\mathrm{P} c b h 1$ to the core region of a strong constitutive promoter Pcdna1 after identifying their function in $T$. reesei. In addition, this work introduced a hybrid promoter for the first time to optimize the $T$. reesei cellulase system by overexpressing two essential cellulases BGLA and EG2. Furthermore, these two cellulases were co-overexpressed under the control of Pcc to improve the saccharification efficiency of $T$. reesei extracellular enzymes cocktail towards two differently pretreated corncob residues. This novel hybrid promoter offered not only a new method in promoter engineering utilized in $T$. reesei, but also limitless possibility to improve the cellulase hydrolysis ability at the transcriptional level.

\section{Results}

\section{Characterization of a series of $5^{\prime}$ truncated promoters of cdna1}

As a strong constitutive promoter in T. reesei, Pcdna 1 has been successfully utilized to express cellulases using glucose as the carbon source [15]. Here, the 928-bp promoter region upstream of ATG of the gene cdna1 was analyzed as shown in Fig. 1. The 
promoter Pcdna 1 contains several core cis-acting elements, such as TATA box, CCAAT box and GC box, which are essential elements to initiate transcription or enhance the transcriptional level [36-38]. To functionally validate the cdna 1 promoter, a promoter library based on Pcdna 1 was established by sequence truncation. Five 5' truncated promoters of cdna 1, Pcdna 1-1 (928 bp), Pcdna1-2 (794 bp), Pcdna1-3 (640 bp), Pcdna 1-4 (490 bp) and Pcdna 1-5 (247 bp), were fused to the reporter gene cbh1 (coding cellobiohydrolase 1 ) and transformed into T. reesei using hph as the selectable marker gene, respectively (Fig. 2a). All these five truncated promoters were capable of directing $\mathrm{CBH}$ activity after culturing with glucose (GMM) or Avicel (AMM, Fig. $2 b$ and c). When glucose was used as the sole carbon source, PD1-2 (cbh1 directed by Pcdna1-2) showed the lowest CBH activity, which was about 0.7-fold of PD1-1 (cbh1 directed by Pcdna 1-1). However, PD1-3 (cbh1 directed by Pcdna1-3) exhibited the highest $\mathrm{CBH}$ activity, which was 1.2-fold higher than that of PD1-1, indicating that the deletion of the 154-bp sequence (-794 to -640 bp) located between Pcdna 1-2 and Pcdna 1-3 enhanced promoter activity. Similar to that under the glucose condition, when the strains were cultured with Avicel, the greatest improvement of $\mathrm{CBH}$ activity was imparted by Pcdna 1-3 (1.4-fold higher than Pcdna 1-1) while the promoter activity of Pcdna 1-2 was relatively weak (0.4-fold lower than Pcdna 1-1), indicating that the cisacting elements facilitating gene expression exist in the 640-bp (-640 to -1 bp) sequence of the promoter (Pcdna1-3). Indeed, three kinds of essential cis-acting elements, CCAAT box, GC box and TATA box, were contained in Pcdna1-3 (Fig. 1a). Thus, Pcdna1-3 could be selected as the high-efficiency core region to initiate gene expression.

\section{Identification of the activation region of the cbh1 promoter}

The promoter of $c b h 1$ is known as the strongest inducible promoter in $T$. reesei, and it contains different types of transcriptional regulator-binding sites [17-21]. It has been reported that several binding sites of transcriptional activators Xyr1, Ace2 and Hap2/3/5 exist in the 248-bp (-869 to -622 bp) regulation region, and three Cre1-binding sites are located in the 48 -bp (-725 to -678 bp) transcriptional repression region [22]. Insertion of the 200-bp (-821 to -622 bp) regulation region without Cre1-binding sites results in significant higher activity than the wild-type promoter [22], demonstrating that the 200-bp (-821 to -622 bp) region of Pcbh1 can serve as an activation region.

Here, the Cre1-binding sites-containing region (-725 to -678 bp) was deleted to construct the modified promoter Pcbh1-dc (821 bp, containing the activation region $\mathrm{AR}$ and the core region $\mathrm{CR}$ ), and the regulation region $\mathrm{RR}$ (-869 to $-622 \mathrm{bp}$ ) was removed from $\mathrm{P} c b h 1$ to construct the core-region promoter Pcbh1-cr (621 bp) as shown in Fig. 3a. To eliminate the interference of native $\mathrm{CBH} 1$, the enhanced green fluorescent protein (eGFP)-encoding gene, egfp, was selected as the reporter gene. Then, the promoters Pcbh1$d c$ and Pcbh1-crwere fused with egfp to construct the corresponding cassettes, respectively (Fig. 3a). The fluorescence intensities of the transformants under the glucose (Fig. 3b) and Avicel (Fig. 3c) conditions were measured, respectively. It was found that the Pcbh1-crtransformant PBC1 showed a barely detectable level of fluorescence intensity, which suggested that the activity of the Pcbh1 core region was relatively weak. On the contrary, the Pcbh1- $d c$ transformant PBD1 displayed remarkable improvement of fluorescence intensity compared to PBC1 under both glucose and Avicel conditions (Fig. 3b and Fig. 3c). Especially, when cultured with Avicel, PBD1 generated a 5.3-fold higher fluorescence intensity than that of PBC1 (Fig. 3c). These results demonstrated that the 200-bp (-821 to -622 bp) region of Pcbh1 could be selected as the high-efficiency activation region to facilitate gene expression.

\section{Construction and assessment of the hybrid promoter $\mathrm{P}$ cc}

As shown above, Pcdna 1-3 possessed the ability to efficiently initiate gene expression, and the 200-bp AR of Pcbh1 was determined to be an enhancer to facilitate the process, which led to the hypothesis that the combination of these two regions may generate a powerful hybrid promoter. In this regard, a novel hybrid promoter Pcc was created by fusing the 200-bp (-821 to -622 bp) activation region of Pcbh1 to the 640-bp (-640 to -1 bp) core region of Pcdna1 (Fig. 4a). To assess the activity of Pcc, the promoter was used to direct the expression of the reporter gene egfp in T. reesei. Meanwhile, the promoters Pcbh1 and Pcdna1 were adopted to direct the expression of egfp as control, respectively. Fluorescence intensity measurement was performed as shown in Fig. 4c. As expected, the transformant PCC (egfp expressed by Pcc) had the highest fluorescence intensity and reached 1.6 and 1.8 times more than the transformants PB1 (egfp expressed by Pcbh1) and PD1 (egfp expressed by Pcdna1) under the Avicel condition, respectively (Fig. 4b). While under the glucose condition, the fluorescence intensity of PCC was comparable to that of PD1 (data not shown). The fluorescence emission detected by fluorescence microscopy showed that the eGFP protein was 
produced successfully in the transformant PCC under both glucose and Avicel conditions (Fig. 4c). These results indicated that the hybrid promoter Pcc could drive gene expression with high efficiency under both repressive and inducible conditions.

\section{Construction of the T. reesei strains overexpressing BGLA directed by PcC}

The $\beta$-glucosidase (BGL) was recognized as one of the bottlenecks of cellulase cocktail in hydrolyzing cellulosic substrates due to its low activity in T. reesei. Thus, the hybrid promoter Pcc was first attempted to direct BGL expression with the aim of optimizing the hydrolysis efficiency of cellulolytic enzyme system. Here, the Aspergillus niger $\beta$-glucosidase gene $b g / A$ was overexpressed by Pcc in T. reesei using pyrG as the selectable marker gene. Two transformants, QPB55 and QPB74, were selected to be cultured on esculin plates to detect their BGL activity using glucose and sodium carboxymethyl cellulose (CMC-Na) as the sole carbon source, respectively (Fig. 5a). The sizes of dark halos of these transformants were much larger than that of the parental strain QP4 no matter on glucose-esculin plates or CMC-esculin plates, which indicated that the overexpression of bgIA driven by Pcc resulted in remarkably improvement of BGL activity. Then, fermentation of these transformants were conducted in GMM and CPM for cellulase production, respectively. SDS-PAGE analysis showed that an approximately 120-kDa band (the expected molecular weight of $A$. niger BGLA) were clearly observed under two conditions in the fermentation broths of QPB55 and QPB74, which were not present in QP4 (Fig. 5b and c). And the bands were determined to be BGLA by MS analysis (data not shown). The transformants QPB55 and QPB74 exhibited the significant increase of BGL activities $(16.6 \mathrm{IU} / \mathrm{mL}$ and $15.1 \mathrm{IU} / \mathrm{mL}$, respectively) when using glucose as the carbon source (Fig. 5d), which was in line with the results observed on esculin plates and SDS-PAGE analysis. Besides, when cultured with Avicel, QPB55 and QPB74 provided 43.5- and 25.7-fold higher BGL activities (27.2 IU/mL and $16.0 \mathrm{IU} / \mathrm{mL})$ than that of the parental strain $(0.6 \mathrm{IU} / \mathrm{mL})$, which led to $85.2 \%$ and $58.6 \%$ enhancements of FPA $(1.6 \mathrm{IU} / \mathrm{mL}$ and $1.4 \mathrm{IU} / \mathrm{mL}$, filter paper activity) in comparison to that of QP4 $(0.9 \mathrm{IU} / \mathrm{mL})$, respectively (Fig. $5 \mathrm{~d}$ and e). These results implied that the overexpression of $b g I A$ driven by the hybrid promoter Pcc remarkedly increased the BGL activity, therefore enhancing the total cellulase activity of $T$. reesei.

\section{Construction of the T. reesei strains overexpressing EG2 directed by Pcc}

The $T$. reesei EG2 has been determined to contribute the most to the endoglucanase activity, suggesting its importance in hydrolysis of cellulosic substrates [6]. Therefore, the endoglucanase gene eg2 was also employed to be overexpressed directed by Pcc in T. reesei using pyrG as the selectable marker gene. Then two transformants, QPE15 and QPE24, were selected and fermented in GMM and CPM, respectively. The SDS-PAGE and MS analysis demonstrated that the EG2 bands in the transformants QPE15 and QPE24 were clearly observed while the band of QP4 was not detected (cultured with glucose) or relatively weak (cultured with Avicel, Fig. 6a and b). Notably, strikingly increased EG activities $(0.3 \mathrm{IU} / \mathrm{mL})$ of these transformants were detected under the glucose condition, whereas EG activity of QP4 was considerably weak (0.06 IU/mL, Fig. 6c). While cultured with Avicel, QPE15 and QPE24 showed $19.1 \%$ and $15.7 \%$ increase of EG activities $(6.2 \mathrm{IU} / \mathrm{mL}$ and $6.0 \mathrm{IU} / \mathrm{mL})$ compared to QP4 $(5.2 \mathrm{IU} / \mathrm{mL})$, leading to 2.5- and 2.0-fold higher FPA (2.1 IU/mL and $1.7 \mathrm{IU} / \mathrm{mL})$ than that of QP4 $(0.9 \mathrm{IU} / \mathrm{mL})$, respectively (Fig. $6 \mathrm{c}$ and d). These results indicated that overexpression of eg2 driven by Pcc remarkedly increased the EG activity, therefore improving the total cellulase activity of $T$. reesei. Based on the observation that the total cellulase activity of $T$. reesei can be improved by overexpressing specific cellulases directed by Pcc, this hybrid promoter was demonstrated to be applied to optimize cellulase system.

\section{Construction of the T. reesei strains co-overexpressing BGLA and EG2 directed by PcC}

Given that the individual overexpression of BGLA and EG2 driven by Pcc could led to improvement of the activities of BGL and EG, respectively, and both of them resulted in the FPA augmentation, a hypothesis was proposed that construction of strains cooverexpressing BGLA and EG2 directed by Pcc could further optimize the T. reesei enzymatic system to improve the cellulolytic ability. To this end, the expression cassette of $b g I A$ driven by Pcc (Pcc-bglA-ptrA) was transformed to EG2-overexpression strain QPE15 using ptrA as the selectable marker gene. Afterwards, two transformants QPEB29 and QPEB70 were selected and verified to successfully express $b g / A$ directed by Pcc (data not shown). Then, QPEB29 and QPEB 70 were cultured in CPM for 7 days at $30^{\circ} \mathrm{C}$ for cellulase production. QPEB29 and QPEB70 showed $2.4 \mathrm{IU} / \mathrm{mL}$ and $2.2 \mathrm{IU} / \mathrm{mL}$ filter paper activities at the end of fermentation, respectively, which were increased by $178.1 \%$ and $156.5 \%$ in comparison to QP4, and were also higher than that of 
QPB55 and QPE15 as expected (Fig. 7). These results indicated that the double overexpression of BGLA and EG2 directed by PcC could generate a more efficient cellulolytic enzyme system than the overexpression of the individual ones in $T$. reesei.

\section{Saccharification of corncob residues by the T. reesei strains co-overexpressing BGLA and EG2 directed by PcC}

The improved total cellulase activities of QPEB29 and QPEB70 led to the presumption that their extracellular cellulase cocktails might perform better than that of the parental strain in the deconstruction of lignocellulosic biomass. To confirm this, the cellulase cocktails produced by the bglA-eg2 co-overexpression strains QPEB29 and QPEB70 were used in the saccharification of acidpretreated corncob residue (ACR) and delignified corncob residue (DCR), respectively. Glucose released in saccharification towards ACR by cellulase cocktails of QPEB29 and QPEB70 were $9.9 \sim 10.5 \mathrm{mg} / \mathrm{mL}$ (corresponding to $28.5 \sim 30.2 \%$ cellulose conversion), which were 2.2- and 2.3-fold higher than that by QP4 (4.5 mg/mL corresponding to $12.9 \%$ cellulose conversion, Fig. 8a). When DCR was used as the substrate, the released glucose detected in the saccharification with QPEB29 and QPEB70 cellulase cocktails reached $31.4 \sim 33.2 \mathrm{mg} / \mathrm{mL}$ (corresponding to $86.0 \sim 91.0 \%$ cellulose conversion), which were 2.2- and 2.4-fold higher than the value of QP4 $(14.0 \mathrm{mg} / \mathrm{mL}$, corresponding to $38.4 \%$ cellulose conversion, Fig. $8 \mathrm{~b})$. These results demonstrated that the double overexpression of BGLA and EG2 directed by Pcc significantly improved the capacity of the T. reesei cellulase system for biomass hydrolysis, implying that this hybrid promoter Pcchas the potential to be utilized as a robust promoter to optimize cellulolytic enzyme system for highly efficient bioconversion of lignocellulosic biomass.

\section{Discussion}

In T. reesei, cost-effective cellulolytic enzyme system is needed to achieve efficient bioconversion of lignocellulosic biomass, and it could be obtained by modifying strains through improving cellulase expression levels and optimizing enzyme system [29, 30]. Promoter development is an essential method for strain modification and metabolic engineering [9]. Thus, this study created a novel hybrid promoter Pcc using the synthetic approach in T. reesei, which enabled higher gene expression than that of the powerful promoter Pcbh1 and Pcdna1.

As there is no software to predict promoter function in $T$. reesei as yet [10], extensive experiments were needed to investigate the ability of promoters. Pcdna1 was generally identified as one of the strongest constitutive promoters in T. reesei, but was not well characterized [10]. Here, Pcdna 1 was truncated from 5' end to construct a promoter library including a series of $5^{\prime}$ deleted promoter fragments. The deletion of a 154-bp sequence (-794 to -640 bp) between Pcdna1-2 and Pcdna 1-3 resulted in an augment (74\% with glucose and $300 \%$ with Avicel) of promoter activity (Fig. $2 \mathrm{~b}$ and c). It was speculated that the binding sites of transcriptional repressors may exist between $-794 \sim-640 \mathrm{bp}$, whose effect on inhibition of gene expression was even stronger than the activation effect of GC box located in this sequence. Besides, deficiency of the 150-bp sequence (-640 to $-490 \mathrm{bp})$ between Pcdna 1-3 and Pcdna 1-4 generated a conspicuous reduction (23\% with glucose and $59 \%$ with Avicel) of promoter activity (Fig. $2 \mathrm{~b}$ and c). Indeed, a CCAAT box is contained in this fragment, and the lack of this cis-element might be responsible for the reduced gene expression intensity. It was reported that the modified $c b h 1$ promoter replacing three Cre1-binding sites to one activator Ace2-binding site and two CCAAT box showed 5.5-fold increase of gene expression while the promoter replacing a Cre1binding site to an Ace2-binding site only exhibited a 1.9-fold increase [21]. In addition, disruption of AAB1, a homolog of Hap5 binding to CCAAT box, enabled an approximately 50\% reduction of am gene expression in Neurospora crassa [39]. These reports indicated that the CCAAT box is an important motif in facilitating gene expression. As a result, it is likely that the absence of the CCAAT box in the promoter Pcdna 1-4 resulted in the depressed promoter strength in this study. Nevertheless, it is also possible that uncertain enhancers were existed in the 150-bp region (-640 to -490 bp) between Pcdna 1-3 and Pcdna 1-4. Collectively, the promoter Pcdna 1-3 containing CCAAT box and maybe unknown enhancers can strongly initiate gene expression and served as a strong promoter.

In the regulation regions of promoters, there exist different transcription factor-binding sites, which comprise of activation sequences and repression sequences [40]. And alteration of these cis-elements would enable diversification of promoter activity $[21,22]$. As the strongest inducible promoter in $T$. reesei, Pcbh1 was widely used in protein production and was well characterized $[16,29,41]$. The transcription factors Xyr1, Ace2 and Hap 2/3/5 complex, whose binding sites present in Pcbh1, were identified to be transcriptional activators in initiating gene expression [19-21]. Therefore, the 200-bp (-821 to -622 bp) activation region of Pcbh1, which contains the binding sites of Xyr1, Ace2 and Hap 2/3/5 complex but without Cre1-binding sites region, behaved as 
an amplifier when fused to the upstream of $\mathrm{Pcbh} 1$ in tandem [22]. Thus, the 200-bp activation region of $\mathrm{P} c b h 1$ was deleted in this study to test its magnitude of amplifying expression. The deficiency of this activation region led to a dramatically decrease in promoter activity, which suggested that this activation region was indispensable to Pcbh1. Meanwhile, the Pcbh1 core region Pcbh1-cr lacking this 200-bp activation region showed a low basal activity (Fig. 3b and c), which raised the possibility that the activity of Pcbh1 could be further improved by replacing the native low-activity core region to a highly efficient one.

Usually, a simple synthetic hybrid promoter requires a minimal core promoter and the defined enhancer [24]. It was found that the abilities of the core sequence and the enhancer of a hybrid promoter were mutually independent, however, both of their strength and combination contribute to the capability of the hybrid promoter [25]. Indeed, based on the strong constitutive promoter $\mathrm{P}_{\mathrm{GPD}}$, a hybrid promoter has been constructed in Saccharomyces cerevisiae using $\mathrm{P}_{\mathrm{GPD}}$ as core region and the upstream activation sequence (UAS) tandem of $\mathrm{P}_{\mathrm{CLB}}$ as the activation region, expanding the transcriptional ability of $\mathrm{P}_{\mathrm{GPD}}$ by 2.5 -fold [26]. It can be concluded that a constitutive promoter could be used as the core region in a hybrid promoter, and in doing so, the promoter activity might be further improved. In this study, to acquire a powerful hybrid promoter, the strong $T$. reesei constitutive promoter Pcdna 1 was first characterized and then the high-efficient region was selected as the core promoter. Meanwhile, the regulation region of $\mathrm{P} c b h 1$ was modified before being selected to serve as enhancer. The hybrid promoter $\mathrm{P} c c$ using the high-efficiency promoter Pcdna 1-3 as core region and the 200-bp (-821 to -622 bp) modified region of Pcbh1 as activation region exhibited an improved protein expression capacity compared with $\mathrm{P} c b h 1$ and $\mathrm{P} c d n a 1$ under Avicel condition, indicating that facilitation of the Pcbh1 activation region was imparted successfully to the Pcdna1 core promoter. However, when glucose was used as sole carbon source, Pcc appeared comparable performance to Pcdna1, which might owe to the cis-elements in the modified activation region of $\mathrm{P} c c$ that could not response to glucose. It has been reported that not all of the promoters with extra UAS elements showed stronger activity in Saccharomyces cerevisiae, especially when more than two UAS elements were added [42]. In addition, Kiesenhofer et al. demonstrated that the inappropriate arrangements and distances of cis-elements have the significant impact on Pcbh1 strength [43]. In this study, the modified activation region containing three more CCAAT boxes inserted into the upstream of Pcdna1 could be regarded as new arrangements of cis-elements, which presumably caused no increase of promoter activity under glucose condition. Nevertheless, high expression of protein was achieved by using this hybrid promoter under Avicel condition, which suggested that $\mathrm{P} c c$ would be utilized to enable cellulase optimization in $T$. reesei.

Due to the relatively low production of BGL in $T$. reesei and the resulted high accumulation of cellobiose that inhibit the cellulase production, BGL was recognized as a bottleneck during the cellulose hydrolysis process [31]. In addition, BGLA of $A$. niger was found to be more efficient in enzymatic hydrolysis of cellulose compared with the native BGL1 in T. reesei on account of its lower adsorption onto lignin $[44,45]$. As a result, $b g / A$ of $A$. niger was selected to be overexpressed under the control of Pcc to enhance the cellulose-hydrolyzing ability of $T$. reesei. In this study, the overexpression of BGLA driven by Pccled to a 43.5-fold enhancement of BGL activity $(27.2 \mathrm{IU} / \mathrm{mL})$ and $85.2 \%$ of FPA $(1.6 \mathrm{IU} / \mathrm{mL})$ compared to the parental strain. It has been reported that 3.7-fold increased BGL activity was obtained by overexpressing bg/1 using a four copy $c b h 1$ promoter [32]. Besides, a modified $c b h 1$ promoter replacing three Cre1-binding sites to two binding sites of transcriptional activator Ace2 and one of Hap2/3/5 complex was also used to overexpress bg/1, which resulted in a 27 -fold augment of BGL activity $(19 \mathrm{lU} / \mathrm{mL})$ and $11 \%$ of FPA compared to the parental strain RUT-C30 [11]. Here, the transformants expressing the A. niger BGLA driven by Pcc showed higher $\beta$-glucosidase activity than the strains in these previous studies, indicating that this chimeric promoter Pcc exhibited prominent $b g / A$-expression capacity. Furthermore, when the transformants were cultured under glucose condition, BGLA could also be detected in a high level with pure extracellular background, which provided an easier and low-cost method to purify BGLA when necessary.

It has been reported that the deletion of EG2 caused a 55\% reduction of endoglucanase activity and a significant decrease of total cellulase activity using lactose as the carbon source, indicating that EG2 was the primary component contributing to the endoglucanase activity and played an important role in cellulose hydrolysis [6]. Here, the EG activity had an $20 \%$ augment by overexpressing eg2 using Pcc in T. reesei, and FPA was increased by $145 \%$ under inducible condition. These results were in agreement with the previous report that the EG2-deletion stain showed an absence of the expression of the other cellulase genes, demonstrating that EG2 plays a pivotal role in forming the inducer of cellulase to hydrolyze cellulose in T. reesei [46]. Collectively, overexpression of EG2 directed by Pcc successfully optimized the cellulolytic enzyme system of $T$. reesei. Besides, when 
transformants cultured under glucose condition, EG2 was also secreted with pure extracellular background (Fig. 6a), which implied that Pcc could also be utilized to simplify and cost down the process to purify the cellulases in $T$. reesei.

In accordance with the previous report that the overexpression of BGL from Penicillium decumbens led to an 8-fold higher BGL activity but only a $30 \%$ increase of FPA [29], the 43.5-fold enhancement of BGL activity in this study only resulted in a $90 \%$ improvement of FPA. Nevertheless, the $20 \%$ increase of EG2 resulted in a 2.5 -fold FPA improvement. These results prompted us to construct strains co-overexpressing BGLA and EG2 activated by Pcc to further improve the enzymatic hydrolysis capability. As expected, the BGLA-EG2 double-overexpressed strain exhibited the significant enhancement in total enzyme activity and biomass saccharification efficiency towards differently pretreated corncob residues, especially against ACR (increased by $130 \%$ ), compared to QP4. In comparison, the Pcbh1-drived overexpression of BGL derived from A. niger and P. decumbens enabled approximately $20 \%$ enhancement of saccharification efficiency using corncob residue and cornstalk as the substrate, respectively [29,33]. Additionally, the overexpression of BGL1 directed by a modified four-copy promoter of Pcbh1 led to a $29 \%$ augment of saccharification efficiency towards corncob residue [32]. Previously, Szijártó et al. identified that EG2 acted as a key component in liquefying pretreated wheat straw [47]. Collectively, the transformants co-overexpressing $b g / A$ and eg2 driven by Pcc in this study exhibited the extraordinary saccharification performance compared to that of the strains individually expressing BGL implied that EG2 might play an important role in liquefaction of pretreated corncob residues as well.

\section{Conclusions}

In this work, a synthetic hybrid promoter Pcc was created and directed high-level expression of the essential cellulase components BGLA and EG2 to achieve the optimized cellulase system in T. reesei. Furthermore, when applied to the saccharification of differently pretreated corncob residues, this cellulolytic enzyme system exhibited the prominent performance that displayed 2.3fold higher saccharification efficiency than that of the parental strain. The chimeric promoter Pcc constructed here was the first hybrid promoter applied in T. reesei, which expanded the promoter engineering toolbox in T. reesei and raised the possibility to generate powerful tunable promoters. Given the results that the strains expressing proteins directed by Pcc in this study did not show growth defects, it seems like the transcription factors were not depleted and this promoter was accommodating to the cellular context in $T$. reesei. Thus, it is possible to tandemly add binding sites of transcriptional activators to the upstream of the promoter to construct more powerful hybrid promoters. Besides, the hybrid promoter strategy raises the possibility of creating desired fine-tuned promoters by combining different enhancers and core promoters with varied magnitudes of strength. Moreover, this synthetic hybrid promoter approach offered a generic strategy to improve cellulolytic enzyme system in bioconversion of lignocellulosic biomass.

\section{Materials And Methods}

\section{Strains, plasmids, cultural conditions and enzyme preparation}

Trichoderma reesei QM53, a strain lacking the mus53 gene required for the nonhomologous end joining (NHEJ) pathway, was used as the host strain in characterization of a series of $5^{\prime}$ truncated promoters of cdna1. The uridine-auxotrophic strain $T$. reesei QP4, constructed from T. reesei QM9414 [48], was used as the control strain for other assays. The plasmids pIG1783 containing the enhanced green fluorescent protein-encoding gene (egfp), pAN7-1 containing trpC terminator ( $\mathrm{Ttr} C \mathrm{C}$ ) and pME2892 containing the ptrA selectable marker gene were employed for gene fragments cloning [49-51]. The plasmid pAB4-1 carriying the $p y r G$ selectable marker gene was adopted for genetic transformation [52]. The fungal strains were cultivated on potato dextrose agar (PDA) plates containing peeled potato extract $200 \mathrm{~g} / \mathrm{L}$, glucose $(2 \%, \mathrm{w} / \mathrm{v})$, agar ( $2 \%$, w/v, Dingguo Corp., Beijing, China; GA010-500

g) for 7 days at $30^{\circ} \mathrm{C}$ to harvest conidia. Then, $10^{8}$ conidia were inoculated in a $500 \mathrm{~mL}$ flask $(200 \mathrm{rpm})$ containing $100 \mathrm{~mL}$ liquid glucose-minimal medium (GMM; MM, [53]) or Avicel-minimal medium (AMM) and incubated at $30^{\circ} \mathrm{C}$. The AMM medium contained $2 \%(\mathrm{w} / \mathrm{v})$ Avicel substituted for $2 \%$ glucose as the sole carbon source and the remaining components of GMM. To produce cellulase, conidia $\left(10^{6} / \mathrm{mL}\right)$ were first inoculated in a $500 \mathrm{~mL}$ flask containing $100 \mathrm{~mL}$ liquid $\mathrm{MM}(1 \%$ glucose) and incubated at $180 \mathrm{rpm}$ and $30^{\circ} \mathrm{C}$ for $36 \mathrm{~h}$ to prepare the seed culture. After that, $10 \mathrm{~mL}$ of the inoculum was transferred into $100 \mathrm{~mL}$ of the cellulase production medium (CPM, [54]) in a $500 \mathrm{~mL}$ flask at $200 \mathrm{rpm}$ and $30^{\circ} \mathrm{C}$. The glucose- and CMC-esculin plates were used to screen the transformants showing $\beta$-glucosidase activity. The medium composition was as follows: $2.0 \mathrm{~g} / \mathrm{L}(\mathrm{NH} 4) 2 \mathrm{SO}$, 
$0.5 \mathrm{~g} / \mathrm{L} \mathrm{MgS04} 7 \mathrm{H} 2 \mathrm{O}, 1 \mathrm{~g} / \mathrm{L} \mathrm{KH} 2 \mathrm{PO} 4,1.0 \mathrm{~g} / \mathrm{L}$ yeast extract, 2\%o (v/v) Triton X-100, $3.0 \mathrm{~g} / \mathrm{L}$ esculin, $0.5 \mathrm{~g} / \mathrm{L}$ ferric citrate, $20.0 \mathrm{~g} / \mathrm{L}$ agar, and $10.0 \mathrm{~g} / \mathrm{L}$ glucose or $10.0 \mathrm{~g} / \mathrm{L}$ sodium carboxymethyl cellulose (CMC-Na) was contained as sole carbon source for glucose-esculin plate or CMC-esculin plate, respectively. The culture medium was supplemented with $0.1 \%$ uracil (Sigma, USA) in the entire research process when necessary.

\section{Construction of strains characterizing P cdna1 and the activation region of Pcbh1}

In this study, expression cassettes were constructed with the method of Double-joint PCR [55]. Primers used in this study were listed in Table 1. Phanta ${ }^{\circledR}$ Super-Fidelity DNA Polymerase (Vazyme Biotech Co., Ltd., Nanjing, China) was used for PCR amplification. To construct the cassettes that a series of 5 ' truncated cdna 1 promoters expressed $c b h 1$, chromosomal DNA of $T$. reesei QM53 was used as the template to amplify five different lengths of 5' deleted Pcdna1 using primer pairs cdna1-1UF (hphR) to cdna1-5UF (hphR)/cdna1-UR (cbh1F), respectively. Upstream homologous arm of $c b h 1$ was amplified using primer pair cbh1UF/cbh1-UR (hphF), and downstream homologous arm cbh1-ORF was amplified using cbh1-F/cbh1-R. The selectable marker hygromycin B gene $(h p h)$ fragment was amplified by hph-F/hph-R. Then the five differently truncated promoters of cdna 1 were respectively employed to fuse with the upstream homologous arm of $c b h 1, h p h$ and $c b h 1$-ORF through Double-joint PCR, and primer pair cbh1-UF/cbh1-R was used to amplify the final cassettes (Fig. 2a). The expression cassettes were purified and transformed respectively into the protoplasts of T. reesei QM53 through the PEG-mediated transformation, which was described previously [53]. The transformants were screened on solid MM containing $300 \mu \mathrm{g} / \mathrm{mL}$ hygromycin B. 
Primers used in this study

\begin{tabular}{|c|c|}
\hline Primers & Nucleotide sequence $\left(5^{\prime}\right.$ to $\left.3^{\prime}\right)$ \\
\hline $\begin{array}{l}\text { cdna1-1UF } \\
\text { (hphR) }\end{array}$ & CCGTCACCAGCCCTGGGTTGCAGACAATGATGGTAGCAGCG \\
\hline $\begin{array}{l}\text { cdna1-2UF } \\
\text { (hphR) }\end{array}$ & CCGTCACCAGCCCTGGGTTGCTCAAACAGCCTCATGGTTCCCAGA \\
\hline $\begin{array}{l}\text { cdna1-3UF } \\
\text { (hphR) }\end{array}$ & CCGTCACCAGCCCTGGGTTGGCCGATTCCTTTCCCGGGATGCTCC \\
\hline $\begin{array}{l}\text { cdna1-4UF } \\
\text { (hphR) }\end{array}$ & CCGTCACCAGCCCTGGGTTGTAGCGAGCGGCCATTTGGAGGTCAT \\
\hline $\begin{array}{l}\text { cdna1-5UF } \\
\text { (hphR) }\end{array}$ & CCGTCACCAGCCCTGGGTTGGCTTGGGTTGGAAGCGATTCCAGGT \\
\hline $\begin{array}{l}\text { cdna1-UR } \\
\text { (cbh1F) }\end{array}$ & ATGACGGCCAACTTCCGATACATGTTGAGAGAAGTTGTTGGATTGAT \\
\hline cbh1-UF & TACGCCACTGTGAGGAGGCC \\
\hline $\begin{array}{l}\text { cbh1-UR } \\
\text { (hphF) }\end{array}$ & TTCAATATCAGTTAAGGTCGTCGGTGAGCCACGTGCTTTTT \\
\hline cbh1-F & ATGTATCGGAAGTTGGCCGTCAT \\
\hline cbh1-R & TTACAGGCACTGAGAGTAGTAAGG \\
\hline hph-F & CGACCTTAACTGATATTGAA \\
\hline hph-R & CAACCCAGGGCTGGTGACGG \\
\hline cbh1-1307UF & AAAGCGTTCCGTCGCAGTAG \\
\hline cbh1-724UR & AAGCCGTTGGCAAATTAC \\
\hline $\begin{array}{l}c b h 1-677 \mathrm{UF} \\
\text { (cbh1-724UR) }\end{array}$ & AAGCCGTTGGCAAATTACCTTCACTCAGTCCAATCTCA \\
\hline $\begin{array}{l}\text { cbh1-UR } \\
\text { (egfpF) }\end{array}$ & CTCGCCCTTGCTCACCATGATGCGCAGTCCGCGGTT \\
\hline cbh1c-UF & GGTGAAGTGAAAGAAGACAGAGGT \\
\hline cbh1-869UF & ACCTGTAAAGCCGCAATG \\
\hline egfp-F & ATGGTGAGCAAGGGCGAG \\
\hline $\begin{array}{l}\text { egfp-R } \\
\text { (TtrpCS) }\end{array}$ & TTTGATGATTTCAGTAACGTTAAGTTTACTTGTACAGCTCGTCCA \\
\hline TtrpC-S & ACTTAACGTTACTGAAATCATCAAA \\
\hline TtrpC-A & GAGTGGAGATGTGGAGTGGG \\
\hline cbh1-621UR & GGAACAAACAAGCGACCC \\
\hline $\begin{array}{l}\text { cdna1-3UF } \\
\text { (cbh1-621UR) }\end{array}$ & GGGTCGCTTGTTTGTTCCGCCGATTCCTTTCCCGGGATGCTCC \\
\hline cdna1-1UF & CAGACAATGATGGTAGCAGCG \\
\hline $\begin{array}{l}\text { cdna1-UR } \\
\text { (cbh1sp) }\end{array}$ & AGCACGAGCTGTGGCCAAGAAGGCCGAGATGACGGCCAACTTCCGATACATGTTGAGAGAAGTTGTTGGATTGAT \\
\hline $\begin{array}{l}\text { cdna1-UR } \\
\text { (egfpF) }\end{array}$ & CTCGCCCTTGCTCACCATGTTGAGAGAAGTTGTTGGATTGATC \\
\hline
\end{tabular}




\begin{tabular}{|c|c|}
\hline Primers & Nucleotide sequence $\left(5^{\prime}\right.$ to $\left.3^{\prime}\right)$ \\
\hline $\begin{array}{l}\text { bglA-F } \\
\text { (cbh1spR) }\end{array}$ & CTTGGCCACAGCTCGTGCTGTACGTGCCGTCACTTCCTT \\
\hline $\begin{array}{l}\text { bglA-R } \\
\text { (Txyn1S) }\end{array}$ & CAACATCAACAGAACCTAGTTGCTTTAGTGAACAGTAGGCAGAGACG \\
\hline $\begin{array}{l}\text { eg2-F } \\
\text { (cbh1spR) }\end{array}$ & СTTGGCCACAGCTCGTGCTCAGCAGACTGTCTGGGGCC \\
\hline $\begin{array}{l}\text { eg2-R } \\
\text { (Txyn1S) }\end{array}$ & CAACATCAACAGAACCTAGTTGCTCTACTTTCTTGCGAGACACGA \\
\hline Txyn1-S & AGCAACTAGGTTCTGTTGATGTTG \\
\hline Txyn1-A & GAATAGCCAGAGGACGGTTG \\
\hline ptrA-S & AACAAAGATGCAAGAGCGG \\
\hline $\begin{array}{l}\text { ptrA-A (cbh1- } \\
\text { 869UF) }\end{array}$ & CCGCTCTTGCATCTTTGTTACCTGTAAAGCCGCAATG \\
\hline
\end{tabular}

The cassettes expressing egfp directed by Pcbh1-dc and Pcbh1-crwere constructed by Double-joint PCR. Firstly, to delete the Cre1binding sites region, two fragments ahead and behind this region were amplified by primer pairs cbh1-1307UF/cbh1-724UR and cbh1-677UF (cbh1-724UR)/cbh1-UR (egfpF), respectively. Secondly, after these two fragments were fused together, the Pcbh1-dc sequence could be amplified using the fused product as the template and cbh1-869UF/cbh1-UR (egfpF) as primer pair. Then, $T$. reesei QP4 genome was used as the template to amplify Pcbh1-cr by primer pair cbh1c-UF/cbh1-UR (egfpF). At the same time, the fragments egfp and trpC terminator were amplified from plasmid pIG1783 and pAN7-1 by prime pairs egfp-F/egfp-R (TtrpCS) and TtrpC-S/TtrpC-A, respectively. Next, the promoter gene fragments Pcbh-dc and Pcbh1-crwere respectively fused with egfp and trpC terminator through Double-joint PCR. Primer pairs cbh1-869UF/ TtrpC-A and cbh1c-UF/ TtrpC-A were used to construct the final cassettes Pcbh1-dc-egfp and Pcbh1-cr-egfp, respectively (Fig. 3a). Finally, the expression cassettes were purified and transformed respectively into the protoplasts of $T$. reesei QP4 together with the plasmid PAB4-1 through the PEG-mediated transformation strategy mentioned above. The transformants were screened on solid MM without pepton.

\section{Generation of promoter P cc and construction of the strains identifying Pcc}

The promoter Pcc was constructed as follows. Firstly, Pcbh1-dc was used as the template to amplify the activation region (AR) of Pcbh1 using primer pair cbh1-869UF/cbh1-621UR. Then, Pcdna1-3 was amplified by primer pair cdna1-3UF (cbh1-621UR)/cdna1UR (egfpF). Subsequently, AR of Pcbh1 was fused to Pcdna1-3 and primer pair cbh1-869UF/cdna1-UR (egfpF) was used to finally amplify the Pcc promoter (Fig. 4a).

After that, Pcc was fused with egfp and TtrpC to get the final expression cassette Pcc-egfp. At the same time, Pcdna 1-egfp and Pcbh1-egfp were constructed as control. Pcdna1 was amplified by primer pair cdna1-1UF/cdna1-UR (egfpF) and then fused with egfp and TtrpC to get the expression cassette Pcdna1-egfp. Pcbh1 was amplified by primer pair cbh1-869UF /cbh1-UR (egfpF) and then fused with egfp and TtrpC to get the expression cassette Pcbh1-egfp. Afterwards, these three cassettes were purified and respectively co-transformed with pAB4-1 into the protoplasts of T. reesei QP4 through the PEG-mediated transformation method mentioned above. The transformants were screened on solid MM without pepton.

\section{Construction of strains overexpressing BGLA or/and EG2 directed by P cC}

To construct the BGLA and EG2 overexpression strains directed by Pcc respectively, the expression cassettes were constructed as follows. The promoter Pcc was amplified by primer pair cbh1-869UF/ cdna1-UR (cbh1sp) using Pcc promoter constructed above as the template. Then the $b g I A$ (GenBank Accession No. AM270402) and eg2 (protein ID: Tr_120312) genes were cloned by primer pairs bglA-F (cbh1spR)/bglA-R (Txyn1S) and eg2-F (cbh1spR)/eg2-R (Txyn1S), respectively. Chromosomal DNA of $A$. niger was used as the template to clone the $\beta$-glucosidase-encoding gene $b g / A$. At the same time, the terminator Txyn 1 was amplified using the template QP4 genome and primer pair Txyn1-S/Txyn1-A. After that, the three fragments, Pcc, bg/A/eg2 and Txyn1 were fused together by Double-joint PCR to get the $b g / A$ and eg2 overexpression cassettes, respectively. Finally, the cassettes were purified 
and transformed together with pAB4-1 respectively into the protoplasts of $T$. reesei QP4 through the PEG-mediated transformation method mentioned above. The transformants were screened on solid MM without pepton.

The BGLA and EG2 co-overexpression strains were constructed by transforming a Pcc-bglA-ptrA cassette to QPE15. The ptrA gene fragment was cloned using the template pME2892 by primer pair ptrA-S /ptrA-A (cbh1-869UF). And the final co-overexpression cassette was constructed by fusing ptrA gene with BGLA overexpression cassette constructed above. The transformants were screened on solid MM with $2 \mu \mathrm{g} / \mathrm{ml}$ of pyrithiamine.

\section{Enzyme activity and SDS-PAGE assay}

The filter paper activity (FPA), cellobiohydrolase (CBH), $\beta$-glucosidase (BGL), and endoglucanase (EG) activities were measured using Whatman no. 1 paper (Whatman, UK), $p$-nitrophenyl- $\beta$-D-cellobioside (pNPC; Sigma, USA), $p$-nitrophenyl- $\beta$-D-glucopyranoside (pNPG; Sigma, USA) and CMC-Na (Sigma, USA) as substrates respectively by the methods described previously [56]. One unit of enzyme activity was defined as the amount of enzyme required to liberate one micromole reducing sugars (FPA, EG) or $p$ nitrophenol ( $\mathrm{CBH}, \mathrm{BGL}$ ) per minute under the assay conditions. SDS-PAGE electrophoresis was performed in $12 \%$ polyacrylamide separating gel with equal volume of culture supernatants boiled for $10 \mathrm{~min}$ for degeneration. The specific cellulase bands were excised for MALDI-TOF-MS identification.

\section{Saccharification of corncob residues}

Acid-pretreated (ACR) and delignified (DCR) corncob residues were used as the substrate in saccharification, and the components of these substrates had been described by Liu et al. [57]. The cellulase loading was 10 IU (FPA)/g substrate equally. The fermentation broths containing $5 \%(\mathrm{w} / \mathrm{v})$ of corncob residue as the substrate were loaded to $30 \mathrm{~mL}$ by adding pH $4.8 \mathrm{citric}$ acid buffer. The $30 \mathrm{~mL}$ reagent was placed in a $100 \mathrm{~mL}$ flask at $150 \mathrm{rpm}$ and $50^{\circ} \mathrm{C}$ for $48 \mathrm{~h}$. Glucose production was detected with an SBA-40C biological sensor analyzer (BISAS, Shandong, China) at the end of saccharification.

\section{Fluorescence and light microscope}

Fluorescence and light microscope (Nikon Eclipse 80i fluorescence microscope) was used to observe the eGFP expression of mycelia. $10^{8}$ spores of the eGFP-expression transformants were inoculated on GMM or AMM plates (GMM or AMM with $4 \mathrm{~g} / \mathrm{L}$ agar) and cultured at $30^{\circ} \mathrm{C}$ for 1 or 4 days. The coverslips were obliquely inserted in the plates at a proper distant from the spores, and the mycelia grown on coverslips was observed directly at the end of cultivation.

\section{Fluorescence intensity measurement}

$10 \mathrm{mg}$ mycelia of the eGFP expression transformant cultured in GMM or AMM was added evenly to $200 \mu \mathrm{L}$ distilled water and

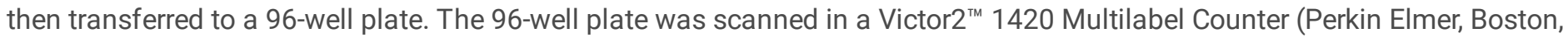
MA) with $485 \mathrm{~nm}$ excitation and $535 \mathrm{~nm}$ emission wavelengths to get the fluorescence intensity.

\section{Abbreviations}

ACR: Acid-pretreated corncob residue; AMM: Avicel-minimal medium; BGL: $\beta$-glucosidase; CBH: Cellobiohydrolase; CCR: Carbon catabolite repression; CMC-Na: Sodium carboxymethyl cellulose; CPM: Cellulase production medium; DCR: Delignified corncob residue; EG: Endoglucanase; FPA: Filter paper activity; GMM: Glucose-minimal medium; pNPC: p-Nitrophenyl- $\beta$-D-cellobioside; pNPG: 4-Nitrophenyl- $\beta$-dglucopyranoside; SDS-PAGE: Sodium dodecyl sulfate polyacrylamide gel electrophoresis; UAS: Upstream activation sequence.

\section{Declarations}

\section{Acknowledgments}

We would like to thank Jingyao Qu, Jing Zhu and Zhifeng Li from State Key laboratory of Microbial Technology of Shandong University for help and guidance in LC-MS. We would also like to thank Caiyun Sun from State Key laboratory of Microbial Technology of Shandong University for help and guidance in Fluorescence intensity measurement. 
YFW and YHZ conceived the work and drafted the manuscript. YFW and RYL performed the experiments and analyzed the data. $\mathrm{XHL}$ and LJS participated in the experiment and collected the data. HL, WCZ, WFL, XS, XML and YHZ designed the work and revised the manuscript. All authors read and approved the final manuscript.

\section{Funding}

This work was supported by the grants from the National Key R\&D Program of China (No. 2018YFA0900503), the National Natural Science Foundation of China (No. 31970070), the Shandong Key Research and Development Program (No. ZR2019ZD19), and the Youth Interdisciplinary Science and Innovative Research Groups of Shandong University (2020QNQT006).

\section{Availability of data and materials}

The data generated or analyzed during this study are included in this published article and its additional information files. Further datasets used and analyzed during the current study are available from the corresponding author on reasonable request.

\section{Ethics approval and consent to participate}

Not applicable.

\section{Consent for publication}

Not applicable.

\section{Competing interests}

The authors declare that they have no competing interests.

\section{References}

1. Bischof RH, Ramoni J, Seiboth B. Cellulases and beyond: the first 70 years of the enzyme producer Trichoderma reesei. Microb Cell Fact. 2016;15(1):106.

2. Seiboth B, Messner R, Gruber F, Kubicek CP. Disruption of the Trichoderma reesei cbh2 gene coding for cellobiohydrolase II leads to a delay in the triggering of cellulase formation by cellulose. J Gen Microbiol. 1992;138(6):1259-64.

3. Fang $\mathrm{H}$, Xia L. High activity cellulase production by recombinant Trichoderma reesei ZU-02 with the enhanced cellobiohydrolase production. Bioresour Technol. 2013;144:693-7.

4. Margolles-Clark E, Harman GE, Penttila M. Enhanced Expression of Endochitinase in Trichoderma harzianum with the $c b h 1$ Promoter of Trichoderma reesei. Appl Environ Microbiol. 1996;62(6):2152-5.

5. Wood TM, McCrae SI. Purification and some properties of a $(1 \rightarrow 4)-\beta$ - d-glucan glucohydrolase associated with the cellulase from the fungus Penicillium funiculosum. Carbohydr Res. 1982;110(2):291-303.

6. Miettinen-Oinonen A, Suominen P. Enhanced production of Trichoderma reesei endoglucanases and use of the new cellulase preparations in producing the stonewashed effect on denim fabric. Appl Environ Microbiol. 2002;68(8):3956-64.

7. Saloheimo M, Kuja-Panula J, Ylösmäki E, Ward M, Penttilä M. Enzymatic properties and intracellular localization of the novel Trichoderma reesei beta-glucosidase BGLII (cel1A). Appl Environ Microbiol. 2002;68(9):4546-53.

8. Uusitalo JM, Nevalainen KM, Harkki AM, Knowles JK, Penttilä ME. Enzyme production by recombinant Trichoderma reesei strains. J Biotechnol. 1991;17(1):35-49.

9. Liu G, Qu Y. Engineering of filamentous fungi for efficient conversion of lignocellulose: Tools, recent advances and prospects. Biotechnol Adv. 2019;37(4):519-29.

10. Fitz E, Wanka F, Seiboth B. The Promoter Toolbox for Recombinant Gene Expression in Trichoderma reesei. Front Bioeng Biotechnol. 2018;6:135. 
11. Li C, Lin F, Li Y, Wei W, Wang H, Qin L, Zhou Z, Li B, Wu F, Chen Z. A ß-glucosidase hyper-production Trichoderma reesei mutant reveals a potential role of cel3D in cellulase production. Microb Cell Fact. 2016;15(1):151.

12. Hägglund P, Eriksson T, Collén A, Nerinckx W, Claeyssens M, Stålbrand H. A cellulose-binding module of the Trichoderma reesei beta-mannanase Man5A increases the mannan-hydrolysis of complex substrates. J Biotechnol. 2003;101(1):37-48.

13. Li J, Wang J, Wang S, Xing M, Yu S, Liu G. Achieving efficient protein expression in Trichoderma reesei by using strong constitutive promoters. Microb Cell Fact. 2012;11:84.

14. Nakari-Setälä T, Penttilä M. Production of Trichoderma reesei cellulases on glucose-containing media. Appl Environ Microbiol. 1995;61(10):3650-5.

15. Uzbas F, Sezerman U, Hartl L, Kubicek CP, Seiboth B. A homologous production system for Trichoderma reesei secreted proteins in a cellulase-free background. Appl Microbiol Biotechnol. 2012;93(4):1601-8.

16. Ilmén M, Onnela ML, Klemsdal S, Keränen S, Penttilä M. Functional analysis of the cellobiohydrolase I promoter of the filamentous fungus Trichoderma reesei. Mol Gen Genet. 1996;253(3):303-14.

17. Takashima S, likura H, Nakamura A, Masaki H, Uozumi T. Analysis of Cre1 binding sites in the Trichoderma reesei cbh1 upstream region. FEMS Microbiol Lett. 1996;145(3):361-6.

18. Saloheimo A, Aro N, Ilmén M, Penttilä M. Isolation of the ace1 gene encoding a Cys(2)-His(2) transcription factor involved in regulation of activity of the cellulase promoter $c b h 1$ of Trichoderma reesei. J Biol Chem. 2000;275(8):5817-25.

19. Aro N, Saloheimo A, Ilmén M, Penttilä M. ACEll, a novel transcriptional activator involved in regulation of cellulase and xylanase genes of Trichoderma reesei. J Biol Chem. 2001;276(26):24309-14.

20. Furukawa T, Shida Y, Kitagami N, Mori K, Kato M, Kobayashi T, Okada H, Ogasawara W, Morikawa Y. Identification of specific binding sites for XYR1, a transcriptional activator of cellulolytic and xylanolytic genes in Trichoderma reesei. Fungal Genet Biol. 2009;46(8):564-74.

21. Zou G, Shi S, Jiang Y, van den Brink J, de Vries RP, Chen L, Zhang J, Ma L, Wang C, Zhou Z. Construction of a cellulase hyperexpression system in Trichoderma reesei by promoter and enzyme engineering. Microb Cell Fact. 2012;11:21.

22. Liu T, Wang T, Li X, Liu X. Improved heterologous gene expression in Trichoderma reesei by cellobiohydrolase I gene ( $c b h 1)$ promoter optimization. Acta Biochim Biophys Sin (Shanghai). 2008;40(2):158-65.

23. Haberle V, Stark A. Eukaryotic core promoters and the functional basis of transcription initiation. Nat Rev Mol Cell Biol. 2018;19(10):621-37.

24. Rushton PJ. What Have We Learned About Synthetic Promoter Construction? Methods Mol Biol. 2016;1482:1-13.

25. Blazeck J, Liu L, Redden H, Alper H. Tuning gene expression in Yarrowia lipolytica by a hybrid promoter approach. Appl Environ Microbiol. 2011;77(22):7905-14.

26. Blazeck J, Garg R, Reed B, Alper HS. Controlling promoter strength and regulation in Saccharomyces cerevisiae using synthetic hybrid promoters. Biotechnol Bioeng. 2012;109(11):2884-95.

27. Gusakov AV, Salanovich TN, Antonov Al, Ustinov BB, Okunev ON, Burlingame R, Emalfarb M, Baez M, Sinitsyn AP. Design of highly efficient cellulase mixtures for enzymatic hydrolysis of cellulose. Biotechnol Bioeng. 2007;97(5):1028-38.

28. Cavaco-Paulo A, Cortez J, Almeida L. The effect of cellulase treatment in textile washing processes. J Soc Dyers Col. 1997;113(7-8):218-22.

29. Ma L, Zhang J, Zou G, Wang C, Zhou Z. Improvement of cellulase activity in Trichoderma reesei by heterologous expression of a betaglucosidase gene from Penicillium decumbens. Enzyme Microb Technol. 2011;49(4):366-71.

30. Zhou J, Wang Y, Chu J, Luo L, Zhuang Y, Zhang S. Optimization of cellulase mixture for efficient hydrolysis of steam-exploded corn stover by statistically designed experiments. Bioresour Technol. 2009;100(2):819-25.

31. Dashtban M, Qin W. Overexpression of an exotic thermotolerant $\beta$-glucosidase in Trichoderma reesei and its significant increase in cellulolytic activity and saccharification of barley straw. Microb Cell Fact. 2012;11:63.

32. Zhang J, Zhong Y, Zhao X, Wang T. Development of the cellulolytic fungus Trichoderma reesei strain with enhanced betaglucosidase and filter paper activity using strong artificial cellobiohydrolase 1 promoter. Bioresour Technol. 2010;101(24):9815-8. 
33. Wang B, Xia L. High efficient expression of cellobiase gene from Aspergillus niger in the cells of Trichoderma reesei. Bioresour Technol. 2011;102(6):4568-72.

34. Suominen PL, Mäntylä AL, Karhunen T, Hakola S, Nevalainen H. High frequency one-step gene replacement in Trichoderma reesei. II. Effects of deletions of individual cellulase genes. Mol Gen Genet. 1993;241(5-6):523-30.

35. Qian Y, Zhong L, Gao J, Sun N, Wang Y, Sun G, Qu Y, Zhong Y. Production of highly efficient cellulase mixtures by genetically exploiting the potentials of Trichoderma reesei endogenous cellulases for hydrolysis of corncob residues. Microb Cell Fact. 2017;16(1):207.

36. Benoist C, Chambon P. In vivo sequence requirements of the SV40 early promotor region. Nature. 1981;290(5804):304-10.

37. Suske G. The Sp-family of transcription factors. Gene. 1999;238(2):291-300.

38. Kato M. An overview of the CCAAT-box binding factor in filamentous fungi: assembly, nuclear translocation, and transcriptional enhancement. Biosci Biotechnol Biochem. 2005;69(4):663-72.

39. Chen H, Crabb JW, Kinsey JA. The Neurospora $a a b-1$ gene encodes a CCAAT binding protein homologous to yeast HAP5. Genetics. 1998;148(1):123-30.

40. Jin LQ, Jin WR, Ma ZC, Shen Q, Cai X, Liu ZQ, Zheng YG. Promoter engineering strategies for the overproduction of valuable metabolites in microbes. Appl Microbiol Biotechnol. 2019;103(21-22):8725-36.

41. Xue X, Wu Y, Qin X, Ma R, Luo H, Su X, Yao B. Revisiting overexpression of a heterologous $\beta$-glucosidase in Trichoderma reesei: fusion expression of the Neosartorya fischeri Bgl3A to $c b h 1$ enhances the overall as well as individual cellulase activities. Microb Cell Fact. 2016;15(1):122.

42. Wang J, Zhai H, Rexida R, Shen Y, Hou J, Bao X. Developing synthetic hybrid promoters to increase constitutive or diauxic shift-induced expression in Saccharomyces cerevisiae. FEMS Yeast Res. 2018;18(8).

43. Kiesenhofer DP, Mach RL, Mach-Aigner AR. Influence of cis Element Arrangement on Promoter Strength in Trichoderma reesei. Appl Environ Microbiol. 2017;84(1):1742-17.

44. Ko JK, Ximenes E, Kim Y, Ladisch MR. Adsorption of enzyme onto lignins of liquid hot water pretreated hardwoods. Biotechnol Bioeng. 2015;112(3):447-56.

45. Lima MA, Oliveira-Neto M, Kadowaki MA, Rosseto FR, Prates ET, Squina FM, Leme AF, Skaf MS, Polikarpov I. Aspergillus niger $\beta$-glucosidase has a cellulase-like tadpole molecular shape: insights into glycoside hydrolase family 3 (GH3) $\beta$-glucosidase structure and function. J Biol Chem. 2013;288(46):32991-3005.

46. Seiboth B, Hakola S, Mach RL, Suominen PL, Kubicek CP. Role of four major cellulases in triggering of cellulase gene expression by cellulose in Trichoderma reesei. J Bacteriol. 1997;179(17):5318-20.

47. Szijártó N, Siika-aho M, Sontag-Strohm T, Viikari L. Liquefaction of hydrothermally pretreated wheat straw at high-solids content by purified Trichoderma enzymes. Bioresour Technol. 2011;102(2):1968-74.

48. Zhong L, Qian Y, Dai M, Zhong Y. Improvement of uracil auxotrophic transformation system in Trichoderma reesei QM9414 and overexpression of $\beta$-glucosidase. CIESC J. 2016;67(6):2510-8.

49. Pöggeler S, Masloff S, Hoff B, Mayrhofer S, Kück U. Versatile EGFP reporter plasmids for cellular localization of recombinant gene products in filamentous fungi. Curr Genet. 2003;43(1):54-61.

50. Punt PJ, van den Hondel CA. Transformation of filamentous fungi based on hygromycin B and phleomycin resistance markers. Methods Enzymol. 1992;216:447-57.

51. Krappmann S, Bayram O, Braus GH. Deletion and allelic exchange of the Aspergillus fumigatus veA locus via a novel recyclable marker module. Eukaryot Cell. 2005;4(7):1298-307.

52. Hartl L, Seiboth B. Sequential gene deletions in Hypocrea jecorina using a single blaster cassette. Curr Genet. 2005;48(3):204-11.

53. Penttilä M, Nevalainen $H$, Rättö M, Salminen E, Knowles J. A versatile transformation system for the cellulolytic filamentous fungus Trichoderma reesei. Gene. 1987;61(2):155-64.

54. Qian Y, Zhong L, Hou Y, Qu Y, Zhong Y. Characterization and Strain Improvement of a Hypercellulytic Variant, Trichoderma reesei SN1, by Genetic Engineering for Optimized Cellulase Production in Biomass Conversion Improvement. Front Microbiol. 2016;7:1349. 
55. Yu JH, Hamari Z, Han KH, Seo JA, Reyes-Domínguez Y, Scazzocchio C. Double-joint PCR: a PCR-based molecular tool for gene manipulations in filamentous fungi. Fungal Genet Biol. 2004;41(11):973-81.

56. Ghose TK. Measurement of cellulase activities. Pure Appl Chem. 1987;59(2):257-68.

57. Liu K, Lin X, Yue J, Li X, Fang X, Zhu M, Lin J, Qu Y, Xiao L. High concentration ethanol production from corncob residues by fed-batch strategy. Bioresour Technol. 2010;101(13):4952-8.

\section{Figures}

a

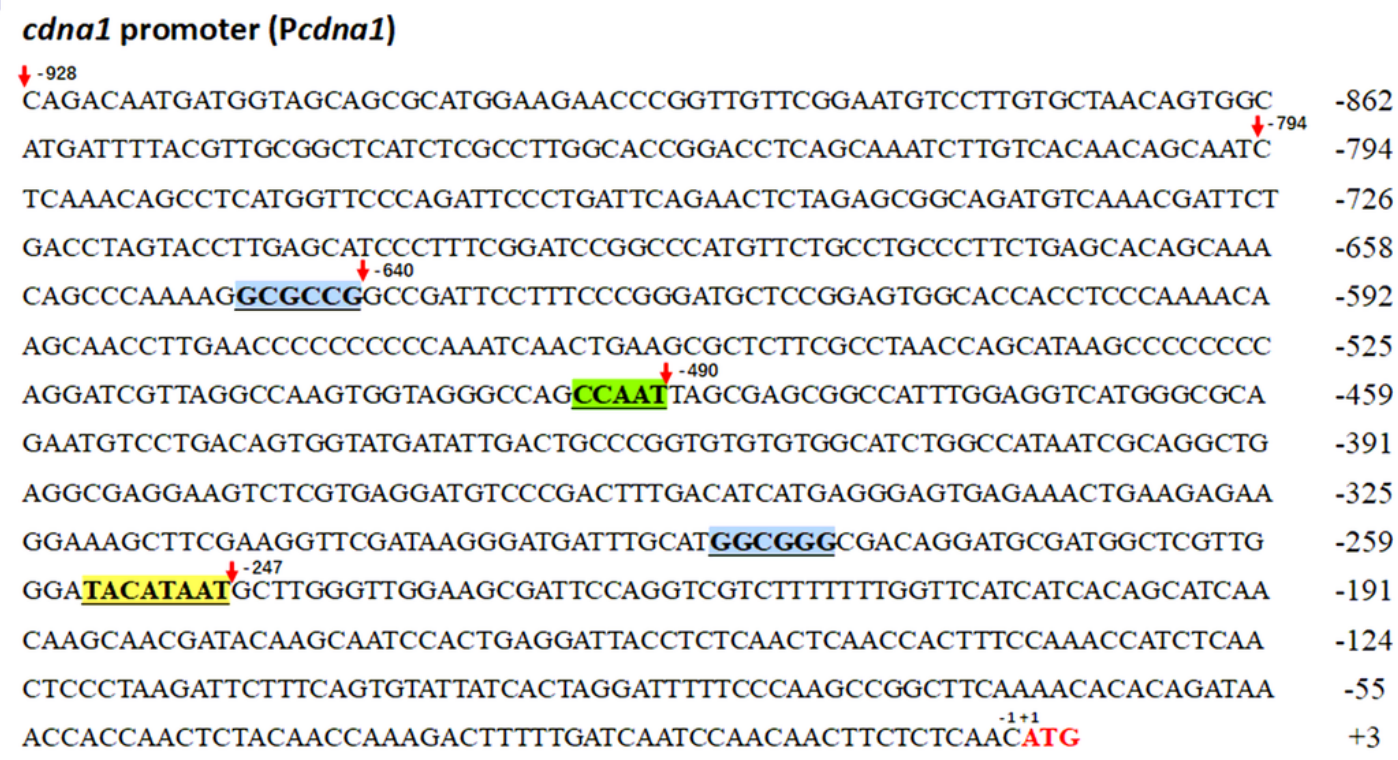

b

cbh1 promoter (Pcbh1)

$\downarrow-869$

ACCTGTAAAGCCGCAATGCAGCATCACTGGAAAATACAAACCAATGGCTAAAAGTACATAAGTTAAT

$-803$

GCCTAAAGAAGTCATATACCAGCGGCTAATAATTGTACAATCAAGTGGCTAAACGTACCGTAATTTGC $\quad-735$

CAACGGCTTGTGGGGTTGCAGAAGCAACGGCAAAGCCCCACTTCCCCACGTTTGTTTCTTCACTCA $\quad$-669

GTCCAATCTCAGCTGGTGATCCCCCAATTGGGTCGCTTGTTTGTTCCGGTGAAGTGAAAGAAGACAG $\quad-602$

AGGTAAGAATGTCTGACTCGGAGCGTTTTGCATACAACCAAGGGCAGTGATGGAAGACAGTGAAATG $\quad-535$

TTGACATTCAAGGAGTATTTAGCCAGGGATGCTTGAGTGTATCGTGTAAGGAGGTTTGTCTGCCGATA $\quad-467$

CGACGAATACTGTATAGTCACTTCTGATGAAGTGGTCCATATTGAAATGTAAGTCGGCACTGAACAGG $\quad-399$

CAAAAGATTGAGTTGAAACTGCCTAAGATCTCGGGCCCTCGGGCCTTCGGCCTTTGGGTGTACATGTT -331

TGTGCTCCGGGCAAATGCAAAGTGTGGTAGGATCGAACACACTGCTGCCTTTACCAAGCAGCTGAGG $\quad-264$

GTATGTGATAGGCAAATGTTCAGGGGCCACTGCATGGTTTCGAATAGAAAGAGAAGCTTAGCCAAGA $\quad$-197

ACAATAGCCGATAAAGATAGCCTCATTAAACGGAATGAGCTAGTAGGCAAAGTCAGCGAATGTGIATA

TATAAAGGTTCGAGGTCCGTGCCTCCCTCATGCTCTCCCCATCTACTCATCAACTCAGATCCTCCAGG $\quad-61$

AGACTTGTACACCATCTTTTGAGGCACAGAAACCCAATAGTCAACCGCGGACTGCGCATCATG + + + +

CCAAT box GC box Xyrl-bingdiing site Are2-bingdiing site Crel-bingdiingsite IATA box

Figure 1

Nucleotide sequences of the Pcdna1 (a) and Pcbh1(b). The nucleotides ahead of A of translation initiation site (ATG) of cdna1 and cbh1 are numbered as - 1 . Known cis-acting elements are shown in different color. The descriptions of elements (TATA box, CAAT box, GC box, Xyr1-binding site, Are2-binding site and Cre1-binding site) are shown in Table S1. An arrow above the sequence indicates the start point of different truncated fragments 
a

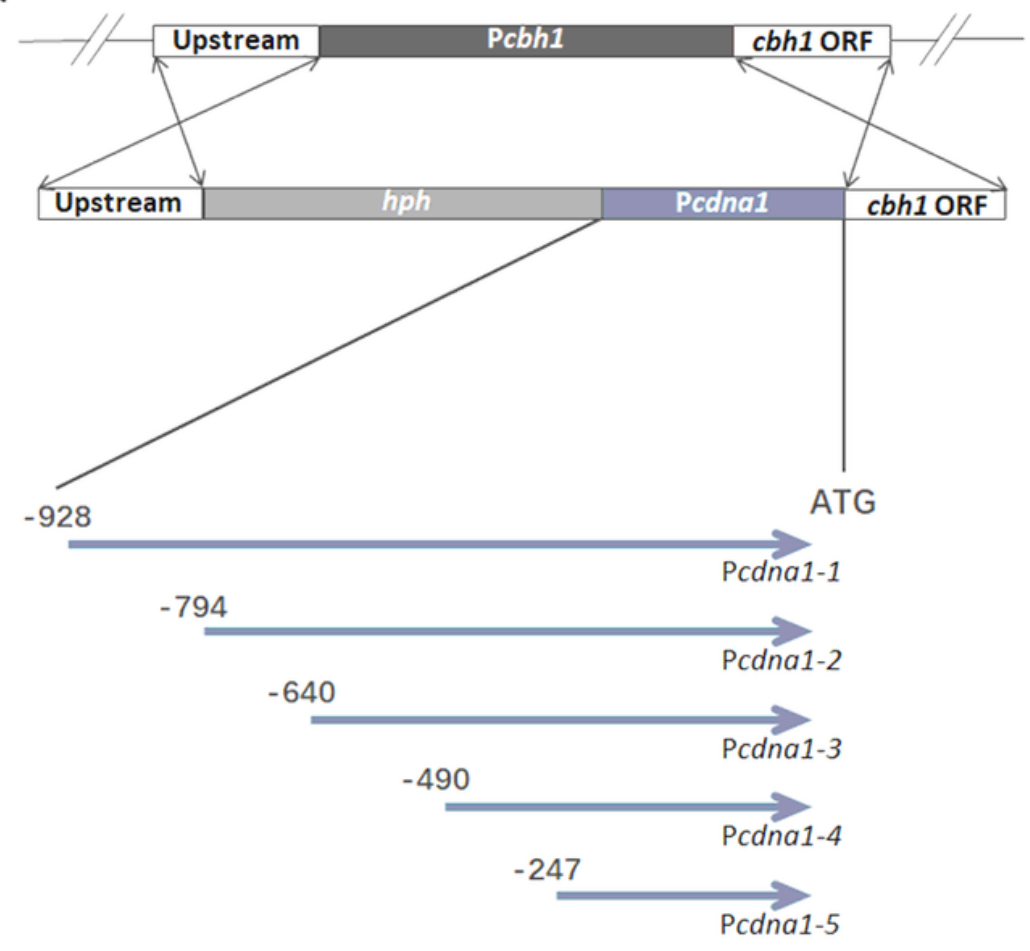

b

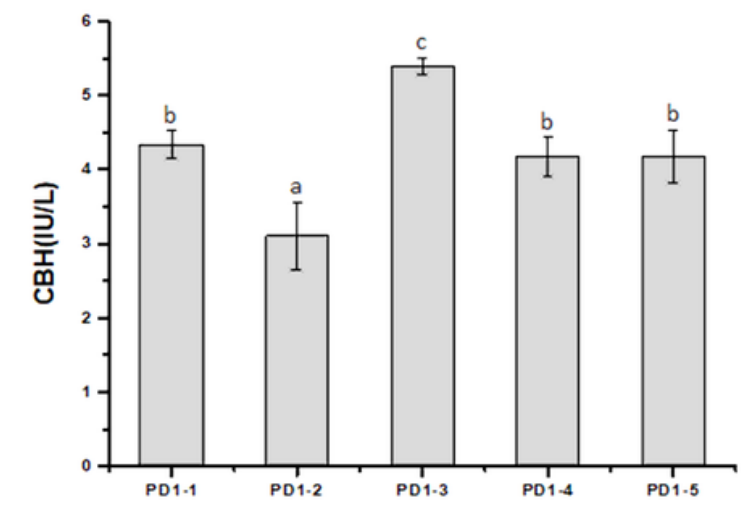

C

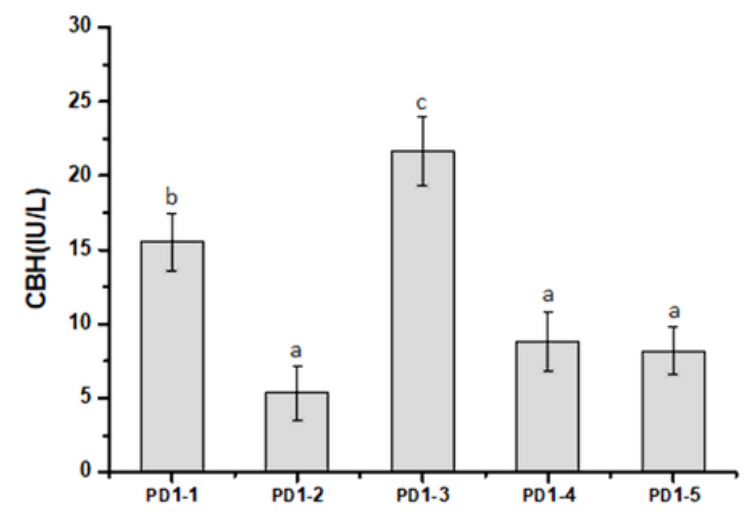

\section{Figure 2}

Identification of the truncated fragments of Pcdna1 fused with cbh1 as the reporter gene. a Strategy of targeted replacement of Pcbh1 with the truncated fragments of Pcdna1 fused with cbh1. b, c CBH activity of the transformants PD1-1 (Pcdna1-1), PD1-2 (Pcdna1-2), PD1-3 (Pcdna1-3), PD1-4 (Pcdna1-4), and PD1-5 (Pcdna1-5) cultured in GMM for 5 days (b) and AMM for 7 days (c). Data indicates the mean of three independent experiments; error bars express the standard deviations. Different lowercase letters above the bars indicate significant differences at $\mathrm{P}<0.05$ 
a

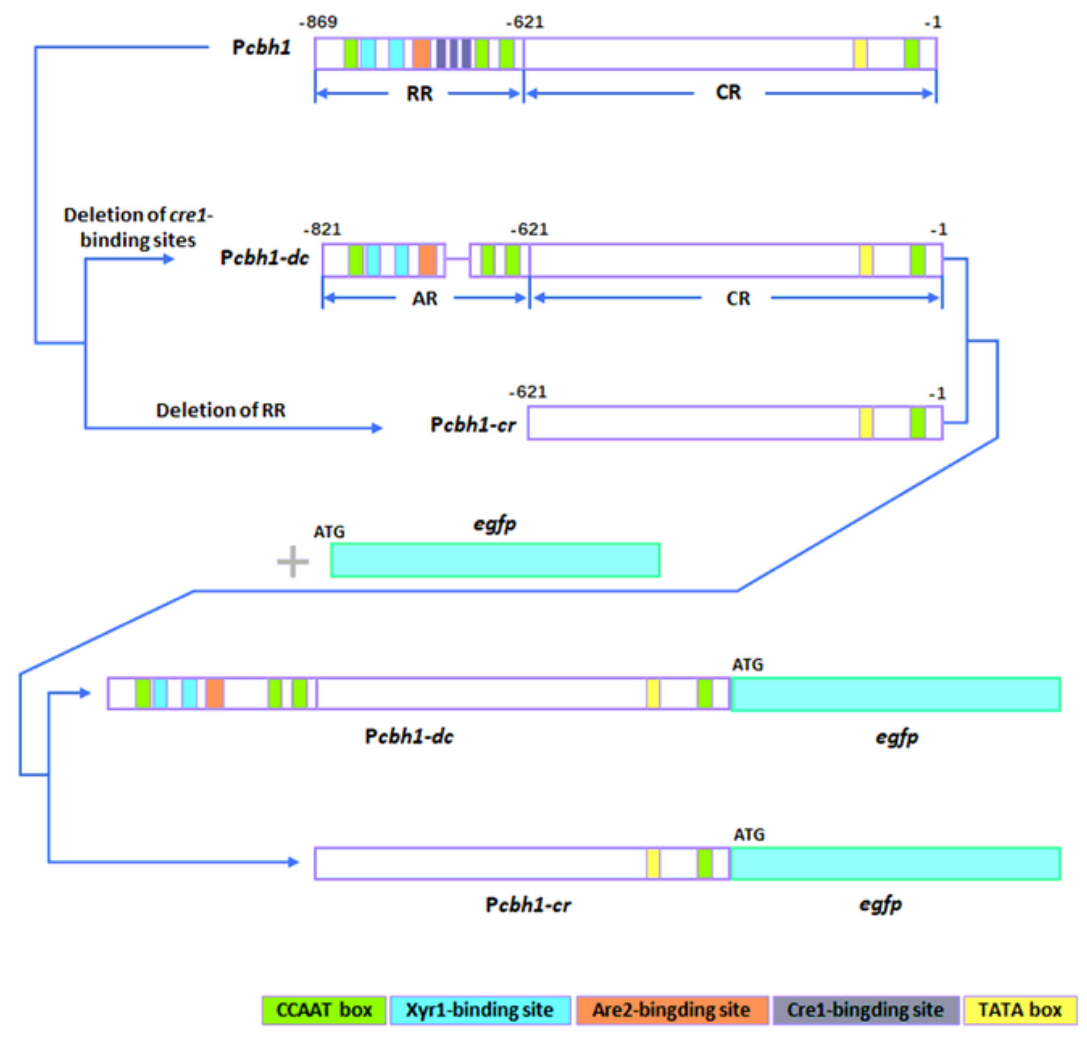

b

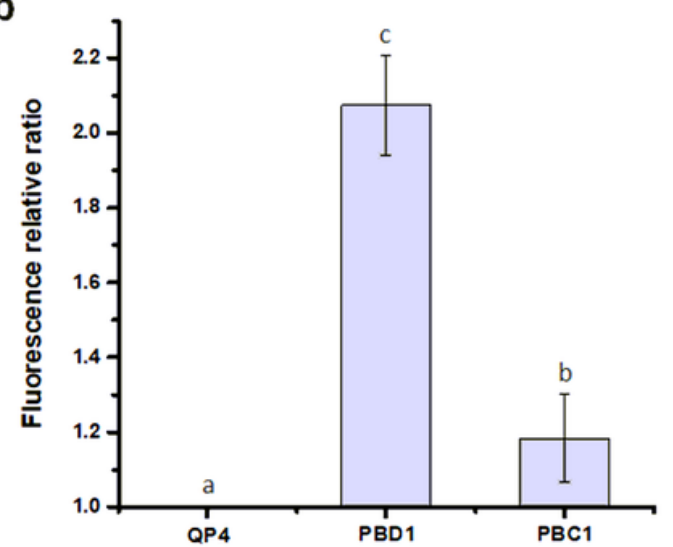

C

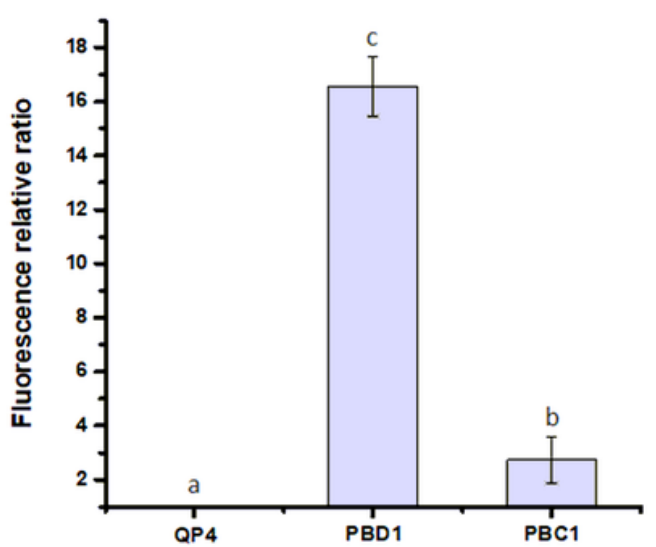

Figure 3

Identification of Pcbh1 activation region (AR) using egfp as the reporter gene. a Strategies of the construction of Pcbh1-dc (Pcbh1 devoid of Cre1-binding sites) and Pcbh1-cr (core promoter of Pcbh1) fused with egfp. RR: regulation region; CR: core region; AR: activation region. b, c Fluorescence relative ratio between the transformants PBD1 (Pcbh1-dc-egfp), PBC1 (Pcbh1-cr-egfp) and the parental strain QP4 cultured in GMM for 2 days (b) and AMM for 3 days (c). Data indicates the mean of three independent experiments; error bars express the standard deviations. Different lowercase letters above the bars indicate significant differences at $\mathrm{P}<0.05$ 
a

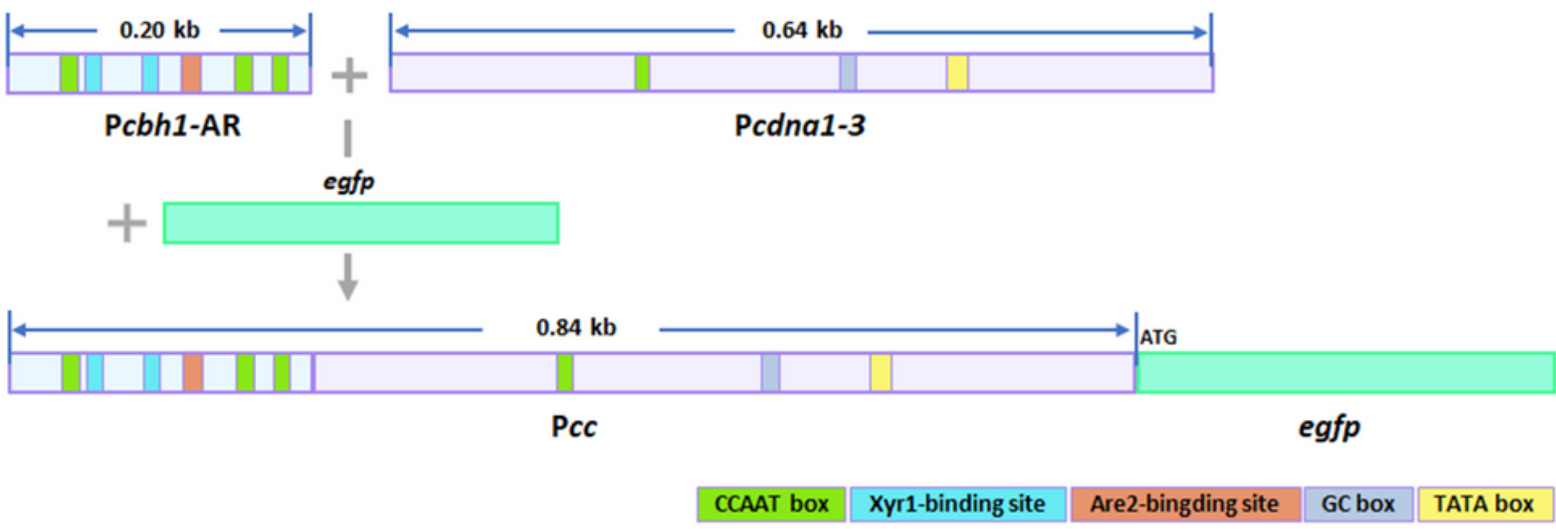

b

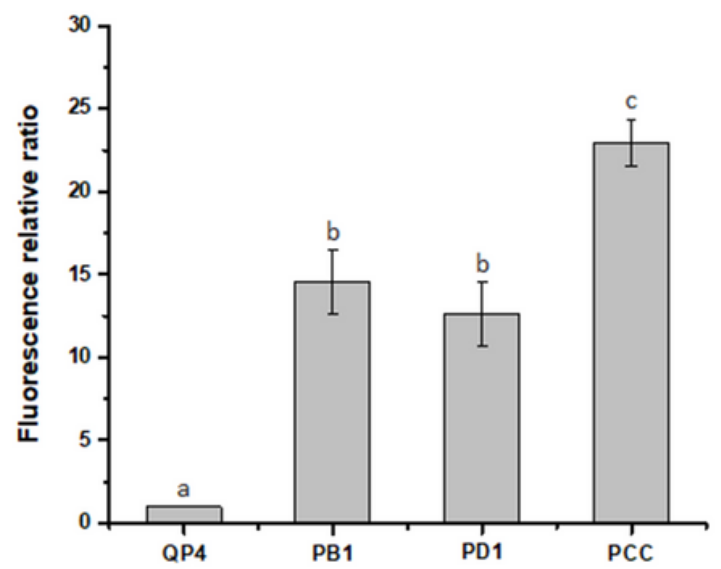

C

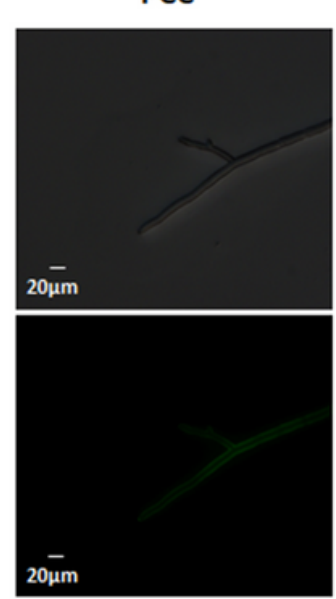

Glucose
PCC

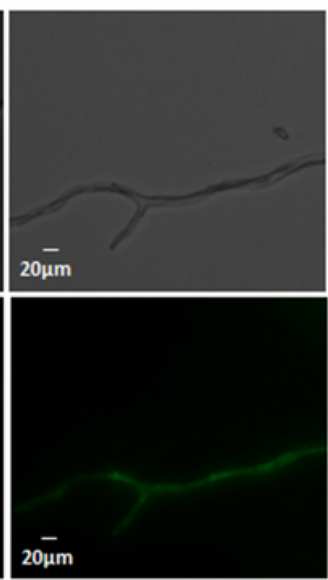

Avicel
QP4

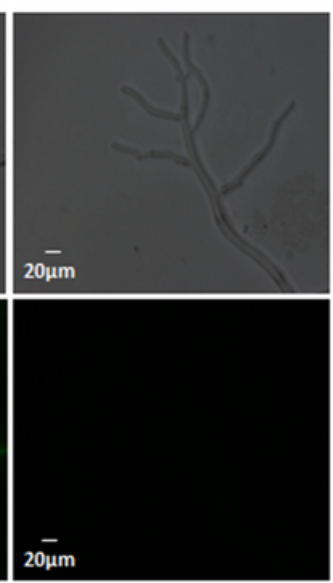

Avicel

\section{Figure 4}

Construction of hybrid promoter Pcc. a Strategies of the construction of Pcc fused with the reporter gene egfp. b Fluorescence relative ratio between the transformants PB1 (Pcbh1-egfp), PD1 (Pcdna1-egfp), PCC and the parental strain QP4, respectively. Strains were cultured in AMM for 2 days. c The fluorescence of transformant PCC (Pcc-egfp) and the parental strain QP4 cultured with glucose for 24 hours and Avicel for 3 days were observed under the fluorescence microscope. The results shown represent one of at least three independent experiments. Data indicates the mean of three independent experiments; error bars express the standard deviations. Different lowercase letters above the bars indicate significant differences at $\mathrm{P}<0.05$ 
a

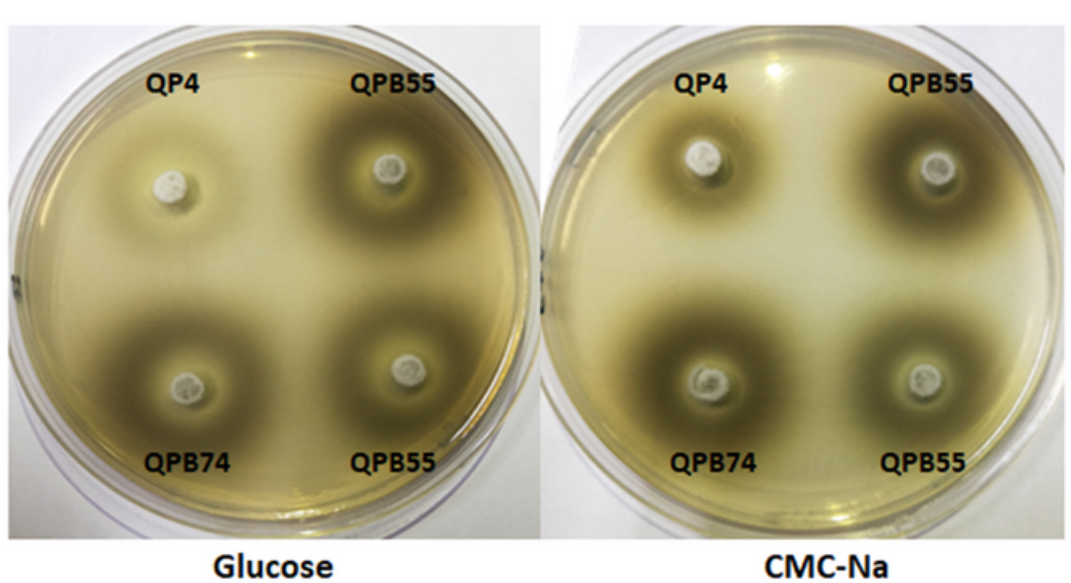

b

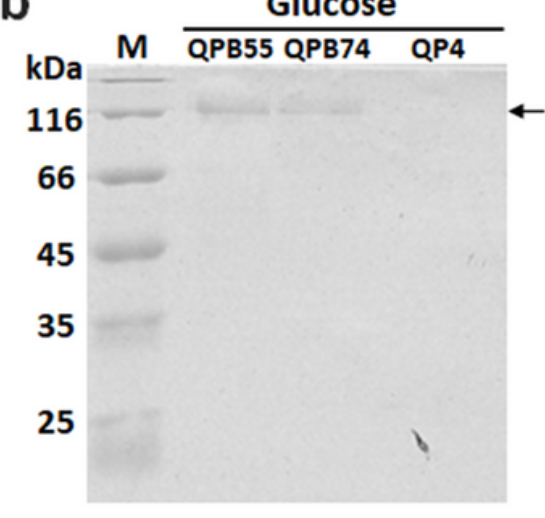

C

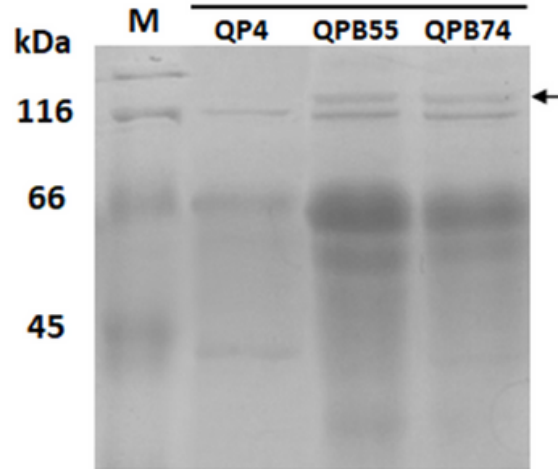

d

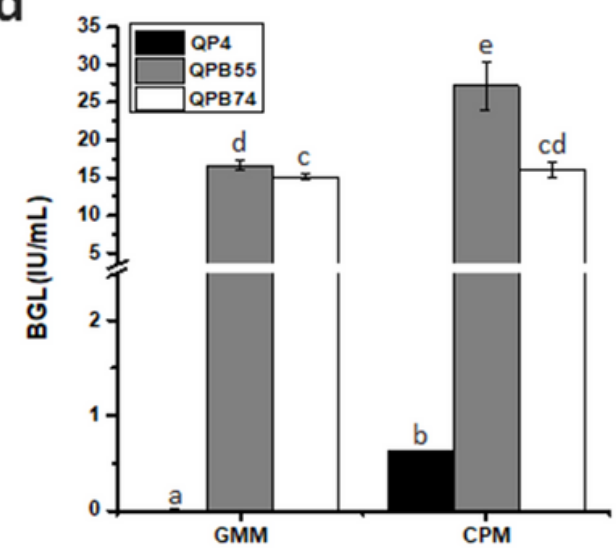

e

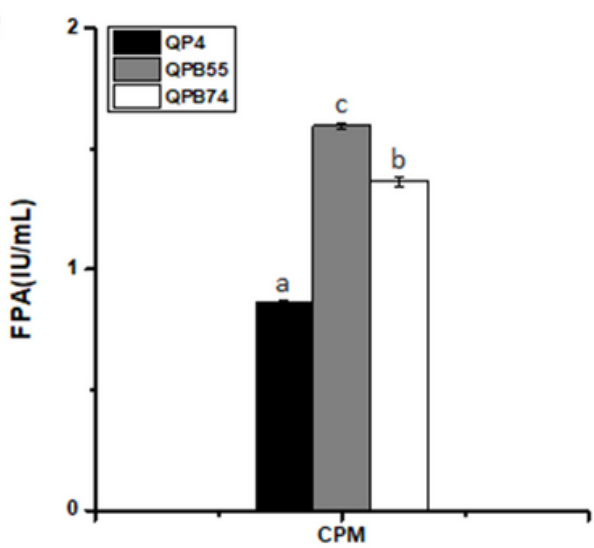

\section{Figure 5}

Expression of bglA under the control of Pcc resulted in increase of BGL activity and FPA. Transformants expressing bglA driven by Pcc and the parental strain QP4 cultured on glucose-esculin plates and CMC-esculin plates at $30^{\circ} \mathrm{C}$ for 2 days (a). SDS-PAGE analysis of the total extracellular cellulases of transformants and QP4 were shown after cultured in GMM for 5 days (b) and CPM for 7 days (c). BGLA bands were indicated by arrows. BGL activity (d) and FPA (e) of the transformants and QP4 were measured after cultured in GMM for 5 days and CPM for 7 days. Data indicates the mean of three independent experiments; error bars express the standard deviations. Different lowercase letters above the bars indicate significant differences at $\mathrm{P}<0.05$ 
a
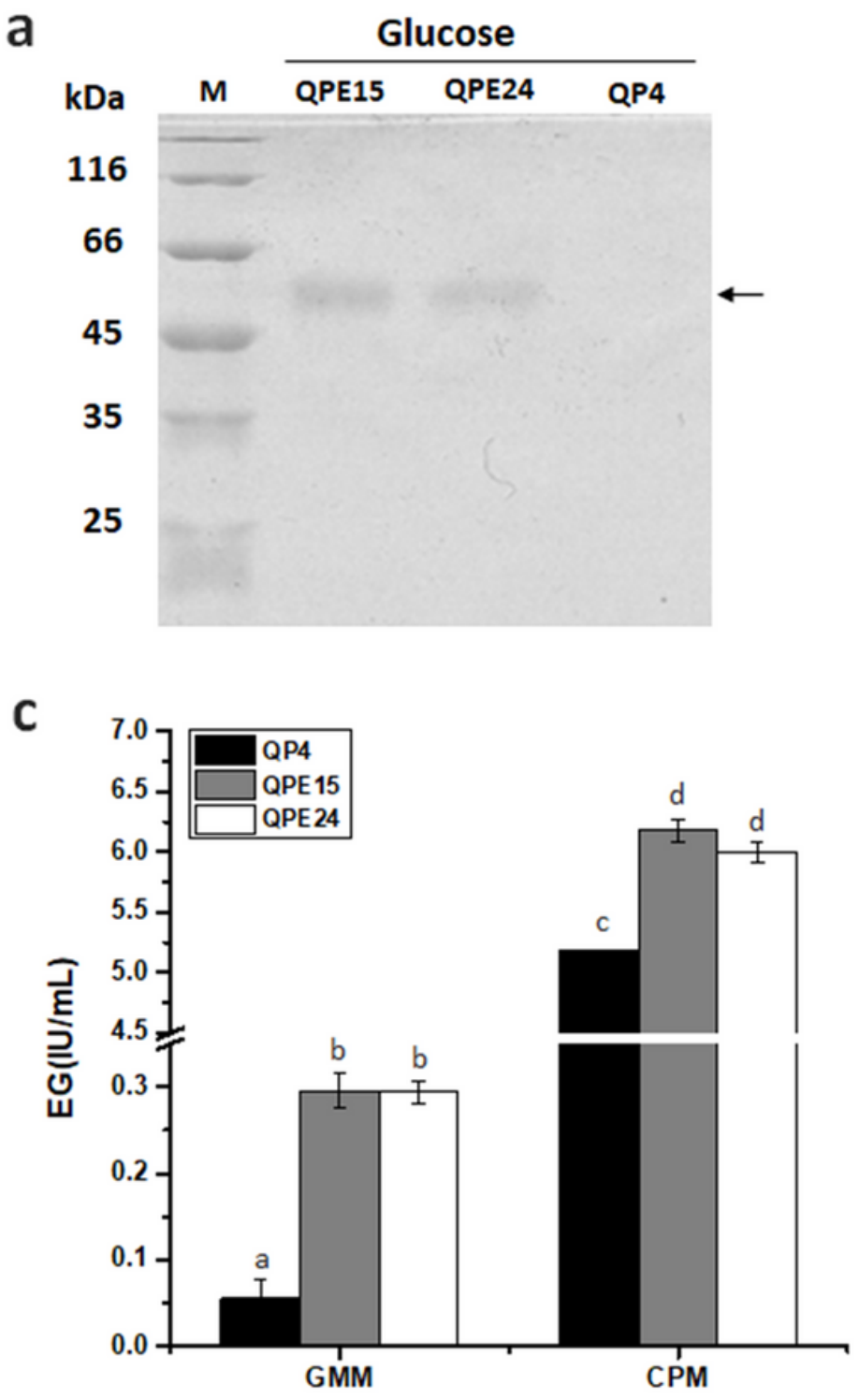

b

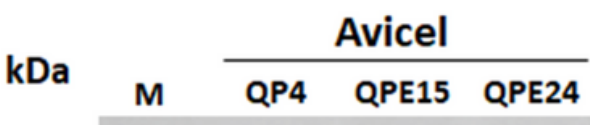

116

66

45

35

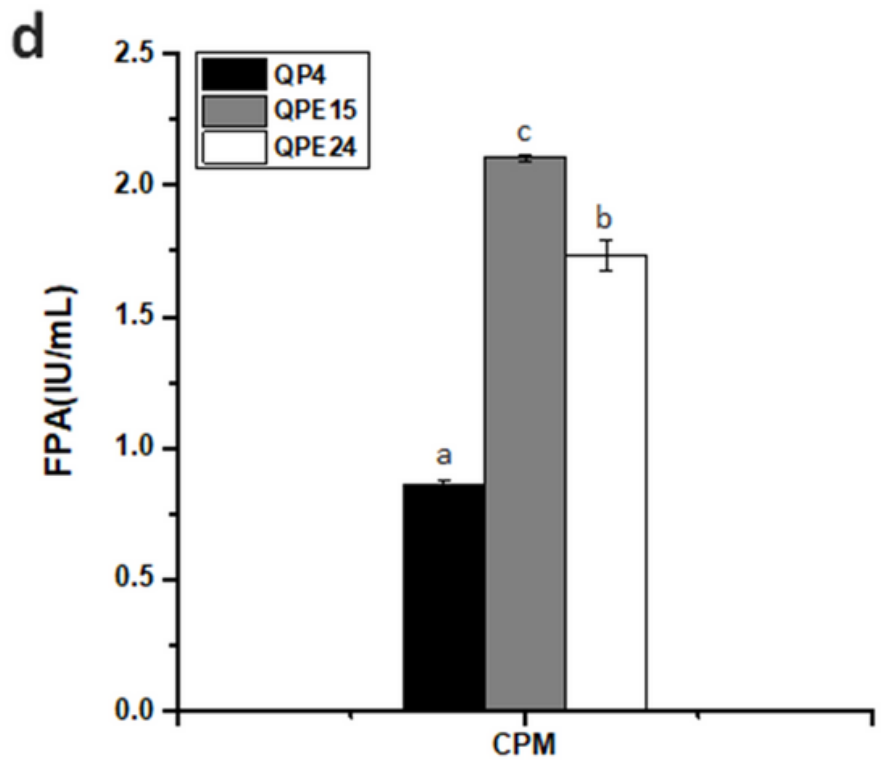

\section{Figure 6}

Expression of eg2 driven by Pcc resulted in increase of EG activity and FPA. SDS-PAGE analysis of the total extracellular cellulases of transformants and the parental strain QP4 were shown after cultured in GMM for 3 days (a) and CPM for 7 days (b). EG2 bands were indicated by arrows. EG activity (c) and FPA (d) of the transformants and QP4 were measured after cultured in GMM for 5 days and CPM for 7 days. Data indicates the mean of three independent experiments; error bars express the standard deviations. Different lowercase letters above the bars indicate significant differences at $\mathrm{P}<0.05$ 


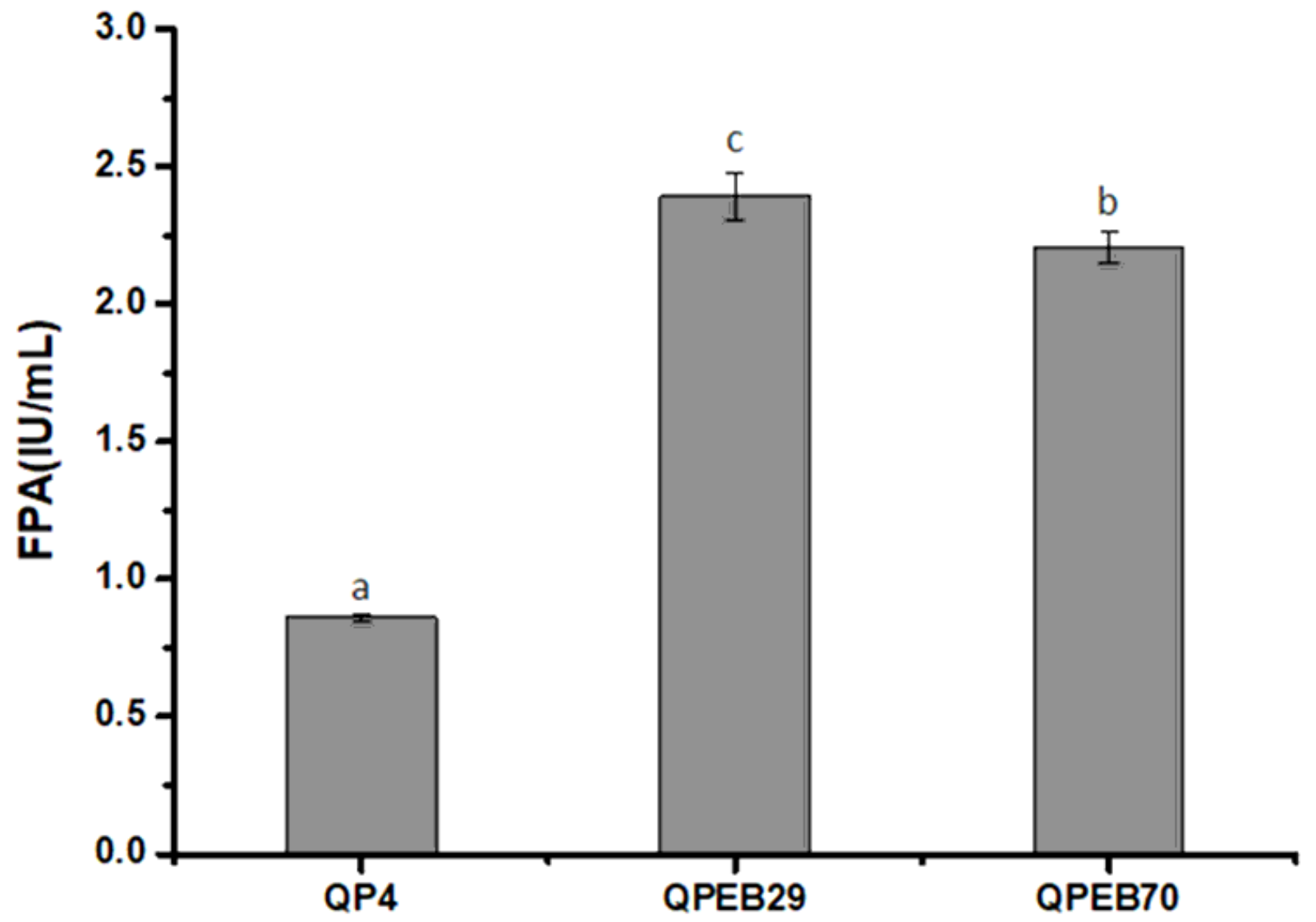

Figure 7

Co-overexpression of bglA and eg2 driven by Pcc in T. reesei resulted in increase of FPA. The transformants and the parental strain QP4 were cultured in CPM for 7 days. FPA were measured at the end of cultivation. Data indicates the mean of three independent experiments; error bars express the standard deviations. Different lowercase letters above the bars indicate significant differences at $\mathrm{P}<0.05$

a

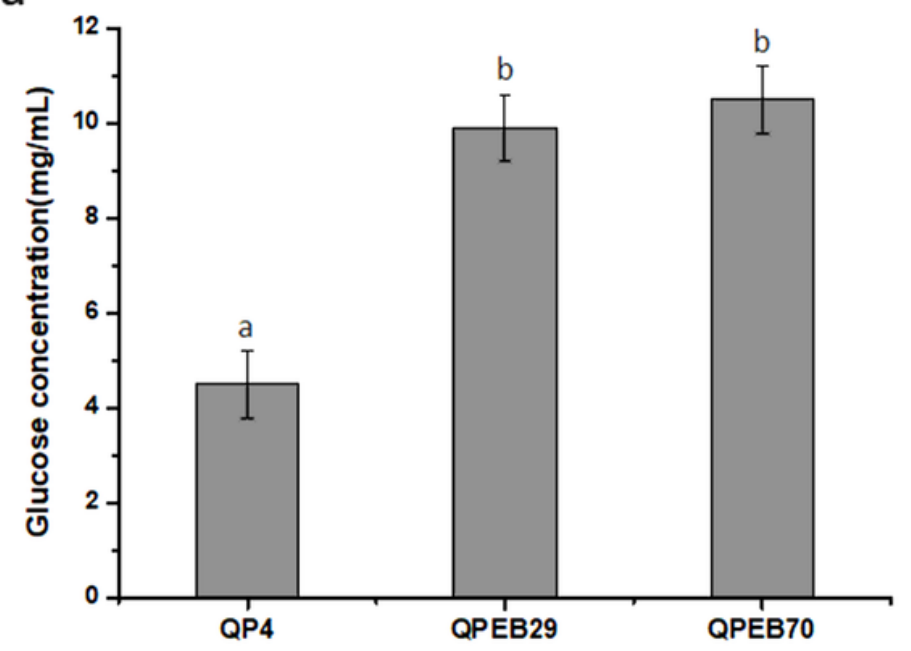

b

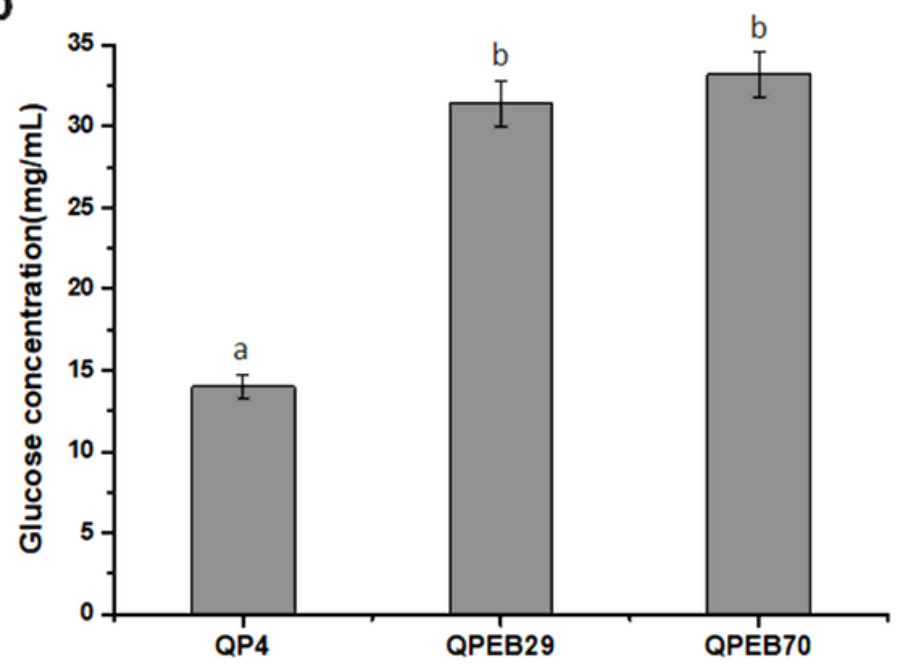

Figure 8 
Saccharification of different pretreated corncob residues using the extracellular enzymes from QP4 and the transformants cooverexpressing bglA and eg2 driven by Pcc. a Glucose released from acid-pretreated corncob residue at 48h. b Glucose released from delignified corncob residue at 48h. Data indicates the mean of three independent experiments; error bars express the standard deviations. Different lowercase letters above the bars indicate significant differences at $P<0.05$

\section{Supplementary Files}

This is a list of supplementary files associated with this preprint. Click to download.

- Tables1.docx 